Neurosurg Focus 28 (2):E2, 2010

\title{
The cerebral sulci and gyri
}

\author{
Guilherme Carvalhal Ribas, M.D. \\ Department of Surgery, University of São Paulo Medical School-LIM-02, Hospital Israelita Albert Einstein, \\ São Paulo, Brazil
}

\begin{abstract}
The aim of this study was to describe in detail the microanatomy of the cerebral sulci and gyri, clarifying the nomenclature for microneurosurgical purposes. An extensive review of the literature regarding the historical, evolutionary, embryological, and anatomical aspects pertinent to human cerebral sulci and gyri was conducted, with a special focus on microneuroanatomy issues in the field of neurosurgery. An intimate knowledge of the cerebral sulci and gyri is needed to understand neuroimaging studies, as well as to plan and execute current microneurosurgical procedures. (DOI: 10.3171/2009.11.FOCUS09245)
\end{abstract}

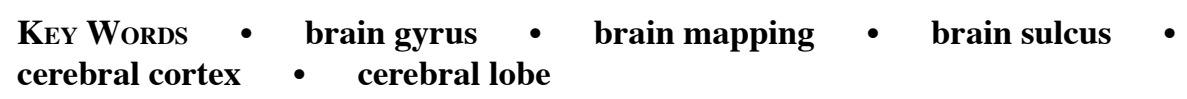

A LTHOUGH there is no strict relationship between brain structure and function, current knowledge shows that the two are closely interrelated. The brain is divided into regions and subdivided into more specific zones, although there is increasing evidence that the borders between those zones are much blurrier than was previously thought. ${ }^{35}$ Therefore, it is essential that neurosurgeons have an intimate knowledge of brain microanatomy, not only to improve their understanding of neuroimaging studies, but also to allow them to plan and perform neurosurgical procedures while considering particular brain functions.

In the relatively new field of microneurosurgery, the development and use of the transcisternal, transfissural, and transsulcal approaches ${ }^{80,84,85}$ have established the sulci as fundamental landmarks on the brain surface. The well-known variability in cortical function ${ }^{1,2,17,47,53,75}$ calls for the aid of cortical mapping techniques to precisely identify specific sites related to cortical function. Nevertheless, detailed knowledge of the structure and form of the cerebral sulci and gyri continues to be mandatory for neuroimaging as well as intraoperative guidance. Once identified, the cerebral sulci can be used by the neurosurgeon either as microneurosurgical corridors or simply as cortical landmarks. ${ }^{61,62}$

A review of the literature regarding the historical, evolutionary, embryological, and anatomical aspects of the cerebral sulci and gyri was conducted to establish detailed descriptions of these structures, as well as their groupings in the brain lobes, for microneurosurgical purposes.

\section{Historical Aspects}

Interest in the human brain dates back to antiquity, and cranial trephination is probably the oldest system- atized surgical procedure. ${ }^{23}$ As early as 10,000 years ago, cranial trephination was performed "successfully" (that is, with new bone formation after the procedure) in the neolithic cultures of Europe, and there are findings dating to 2000 years ago in South America, where the practice was particularly common in the pre-Incan and Incan cultures of Peru. ${ }^{26}$ Despite this historical context, knowledge of the anatomy of the brain in general and of its surface in particular is quite recent. ${ }^{23,26,38}$

The first significant contributions to neuroanatomy were made during the Golden Age of Greek civilization. Hippocrates (460-370 BC), who is considered the father of medicine, posited that the brain was responsible for mental activities and convulsions, whereas some important Greek philosophers, such as Aristotle (384-322 BC), believed that the heart was the seat of intellectual, perceptual, and related functions. ${ }^{23}$

In Alexandria, Egypt, then a Greek city that was particularly advanced in cultural terms, human dissections, which had until then been forbidden in Greek society, began to be performed in $\sim 300 \mathrm{BC}$. There, Herophilus (ca. 335-280 BC), a follower of Hippocrates and considered the father of anatomy, studied the brain and its ventricles as well as the cerebellum, distinguished the motor nerves from sensory nerves, and described the confluence of the cranial venous sinuses, whose name was originally derived from his own name ("torcular herophili"). One of his contemporaries and compatriots, Erasistratus (ca. $310-250 \mathrm{BC}$ ) studied the comparative anatomy of the brain surface and had already hypothesized that there was a relationship between intellect and gyral complexity. ${ }^{23}$

Despite the importance of the Greek contribution, the most widely known anatomical descriptions of classical antiquity are those of Galen (130-199 AD), who studied 

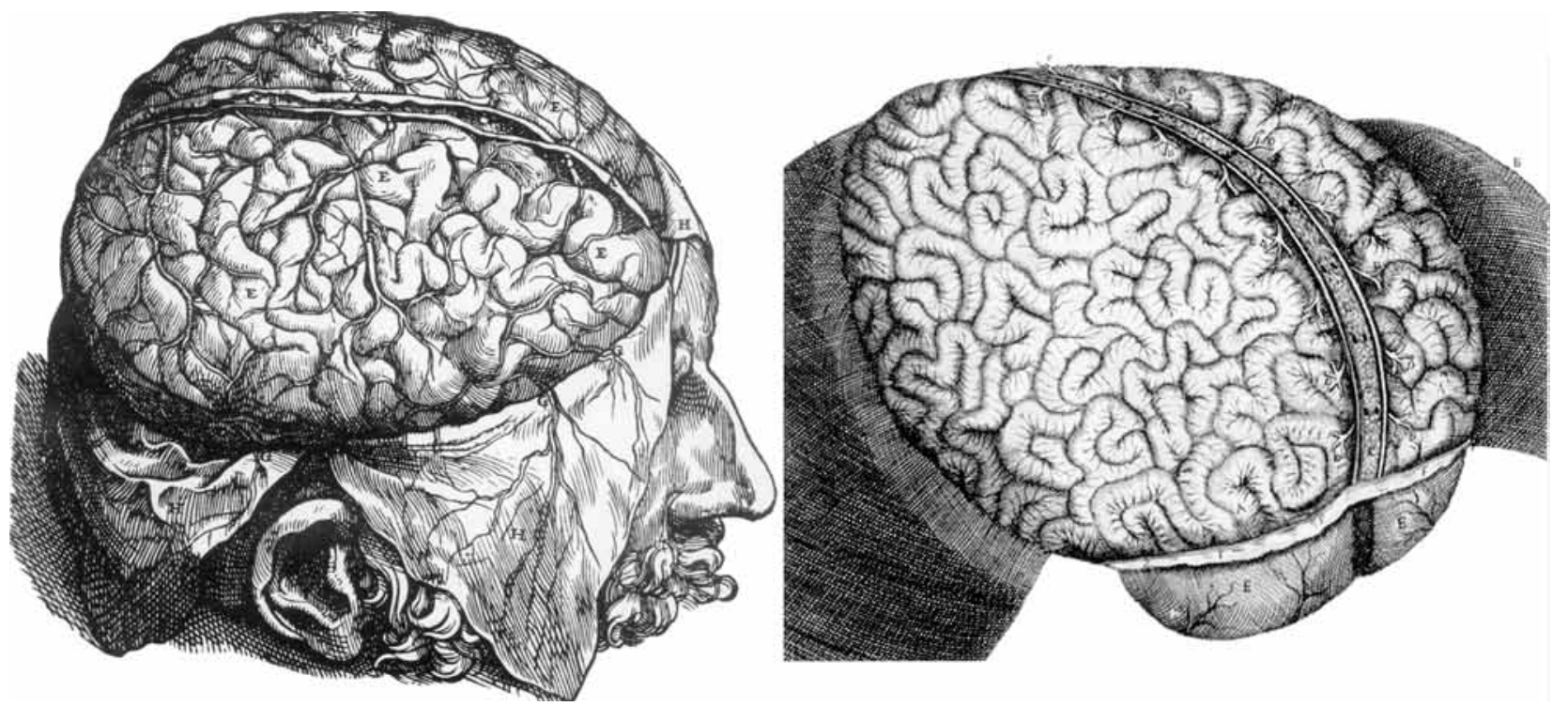

FIG. 1. Renaissance illustrations of the brain sulci and gyri with a configuration that resembles the small bowel disposal. Left: By Andreas Vesalius, 1543. ${ }^{65}$ Right: By Raymond Vieussens, 1684. Reprinted from Vieussens R: Nevrographia Universalis. Frankfurt: Georgium Wilmelmum Künnium, 1690.

anatomy in Alexandria, returned to Greece, and finally settled in Rome, where he was surgeon to the gladiators and performed dissections, primarily on animals. ${ }^{38}$

The Middle Ages, roughly from the 4th to the 14th century, is well known to have been poor in terms of scientific developments in general. Although there were some contributions from individuals in certain regions-in the Arabic world, from Avicenna (980-1037 AD), who some authors credit with the first representation of the brain, made in $\sim 1000 \mathrm{AD}^{69}$ and in Europe, from Mundino dei Luzzi ${ }^{69}$ who, in 1316, performed the first human dissections reported in Europe-anatomical studies were quite limited, principally because of the prohibition against the dissection of human cadavers. During the Renaissance, this prohibition was finally lifted, which led to the progressive development of all anatomical knowledge. The most preeminent figure in this field was undoubtedly Andreas Vesalius (1514-1564), professor of anatomy and surgery at the University of Padua, who published the textbook $D e$ Humanis Corporis Fabrica ${ }^{65}$ in which he pointed out the many errors made by Galen, outlined the distinctions between white matter and gray matter, and described various other aspects of brain anatomy. ${ }^{23,38,69}$

Although Vesalius provided intricate anatomical descriptions, especially of the ventricular cavities and their related deep neural structures, illustrations of the brain convolutions, including those of Vesalius, continued to show them in a chaotic arrangement. Among other notable Renaissance authors were the great Leonardo da Vinci (1472-1519), who, in addition to his elegant descriptions of the brain ventricles, created beautiful illustrations of the brain surface, ${ }^{12}$ and Julius Casserius (ca. 1545-1616), who depicted the brain convolutions, which were at that time understood to resemble the small bowel (Fig. 1). ${ }^{69}$

In 1663 Franciscus de la Böe (1614-1672), also known as Dr. Sylvius, described the lateral cerebral sulcus, which therefore came to be known as the sylvian fissure. ${ }^{23}$

In 1664 Thomas Willis (1621-1675) published his highly regarded Cerebri Anatome, which featured illustrations by the renowned architect Christopher Wren (1632-1723). In addition to describing the group of arteries surrounding the base of the brain (now known as the circle of Willis), Willis introduced a variety of terms, including neurology, hemisphere, lobe, corpus striatum, peduncle, and pyramid, and showed a relationship between the cerebral gyri and memory. ${ }^{23}$

Raymond Vieussens (1644-1716) published the famous Neurographia Universalis in $1690,{ }^{76}$ describing in detail the "centrum semiovale" and other cerebral structures but still illustrating the brain surface similarly to the small bowel. ${ }^{23,69}$ Godefroid Bidloo clearly displayed the central sulcus in his atlas and textbook published in $1685,{ }^{69}$ and subsequently Félix Vicq d'Azyr (1748-1794), famous for describing the mamillothalamic tract, also described the precentral and postcentral convolutions and coined the term uncus $^{69}{ }^{6}$ Later, Johann Christian Reil (1759-1813) provided a comprehensive description of the insula, which had been identified by Bartholin in 1641.23,69 In 1827 Herbert Mayo, student of the renowned anatomist and surgeon Charles Bell (1774-1842), published illustrations of the corona radiata and the internal capsule as well as other important tracts. ${ }^{74}$ In 1829 , the Italian anatomist Luigi Rolando (1773-1831) published his text Della Strutura degli Emisferi Cerebrali, ${ }^{33}$ becoming the first author to accurately portray the cerebral sulci and convolutions, including the central sulcus, which later received his name and is still occasionally referred to as the fissure of Rolando. ${ }^{23,74}$

It was the German physiologist Friedrich Arnold (1803-1890) who first used the terms frontal, parietal, and occipital to describe the cranial bones. In a text published in $1851,{ }^{5}$ Arnold recognized only the sylvian fissure and the parietooccipital sulcus (then known as the internal perpendicular fissure) as anatomically constant sulci. He did not recognize any organized arrangement among the cerebral gyri, and he described the temporal region as an anterior extension of the occipital region. 
The cerebral sulci and gyri

TABLE 1: Mammalian cortical development*

\begin{tabular}{|c|c|c|}
\hline $\begin{array}{l}\text { Primitive Cortex: Allocortex } \\
\text { (3 layers) }\end{array}$ & $\begin{array}{l}\text { Medial Cortical Ring: Mesocortex } \\
\text { (6 layers) }\end{array}$ & $\begin{array}{l}\text { Lateral Cortical Ring: Isocortex } \\
\text { (6 organized layers) }\end{array}$ \\
\hline limbic structures & paralimbic structures \& insula & parainsular \& other neocortical structures \\
\hline archicortex & \multicolumn{2}{|c|}{ neocortex } \\
\hline amygdala & \multirow{3}{*}{ cingulate gyrus } & motor cortex \\
\hline hippocampus & & sensory cortex \\
\hline paleocortex & & visual cortex \\
\hline \multirow[t]{3}{*}{ olfactory or piriform cortex } & \multirow[t]{3}{*}{ insula } & auditory cortex \\
\hline & & language cortices (humans) \\
\hline & & rest of neocortex, associative areas \\
\hline
\end{tabular}

* Adapted from Sarnet and Netsky.

The French anatomist Louis Pierre Gratiolet (1815$1865)$ provided the first accurate descriptions of the cerebral lobes and cerebral fissures. ${ }^{6,72,74}$ In addition to his well-known description of the optic radiation, Gratiolet also distinguished between primary and secondary sulci based on their phylogenetic appearance and adopted the terms initially proposed by Arnold to divide each cerebral hemisphere into 5 lobes (frontal, parietal, occipital, temporal, and insular). Gratiolet coined the elegant term plis de passage to describe the connections between adjacent gyri. He was the first anatomist to understand and describe the fact that despite individual variations, the cerebral sulci and gyri are organized in accordance with a general arrangement. ${ }^{27,74}$ It is notable that despite the intense interest that humankind has always had in relation to the brain, it was only in the middle of the 19th century that the anatomical organization of the cerebral sulci and gyri was perceived and described.

The work of Gratiolet laid the foundation for the important studies later conducted by the French anatomist, anthropologist, and surgeon Paul Broca (1824-1880), who examined the cerebral sulci and fissures. Broca was the first to describe the craniocerebral topographical relationships ${ }^{6,7}$ and to outline the motor speech area of the brain. ${ }^{4}$ In 1861 Broca introduced the concept of cerebral localization. ${ }^{7,36}$ Using as a basis the correlations he found between cranial points and the brain surface, ${ }^{3,5}$ Broca became a pioneer of modern neurosurgery, establishing original guiding anatomical landmarks. ${ }^{3}$

In 1869 Alexander Ecker accurately described all of the cerebral sulci and gyri, introducing the designations orbital, precentral, parietooccipital, and transverse occipital to describe the various sulci. ${ }^{69}$ William Turner (1832-1916) also studied the cerebral sulci in detail, and Turner's sulcus became an eponym for the intraparietal sulcus. ${ }^{36}$

Brodmann in $1909^{9}$ and Von Economo in $1925^{77}$ studied the cerebral sulci and gyri in greater detail, describing the cytoarchitectonic features of the gyri.

\section{Evolutionary Aspects}

According to evolutionary theory, the primitive fishes left the oceans $\sim 350$ million years ago. ${ }^{24}$ The transition from fish to amphibian to reptile involved profound physical transformations, one of which was related to the CNS, which then consisted of a spinal cord segment with an incipient brainstem, hypothalamus, and striatum. The CNS evolved to include the olfactory lobes needed to perceive the new world, as well as the hippocampus and amygdala, which, in conjunction with the hypothalamus, would allow them to analyze their new perceptions and direct their behavior. ${ }^{10,41,64,67}$ Since these new structures surrounded the top of the primitive CNS, they were called limbic, from limbus, the Latin word for ring. ${ }^{22}$ To create different input and output connections, their cells were organized in a laminar arrangement, characterizing then the most primitive cortices, denominated the archicortex (amygdala and hippocampus) and paleocortex, the latter also known as the olfactory (piriform) cortex. ${ }^{10,64}$ The development of the cerebral hemispheres and their cortical surfaces, in particular, began soon after the appearance of these primitive structures. The neocortex first appeared in the brains of early mammals ( 230 million years ago) and became more complex throughout the evolution of the primates, culminating $\sim 50,000$ years ago with the emergence of modern humans..$^{10,24,25}$

According to Sarnat and Netsky, ${ }^{64}$ cytoarchitectonic evidence suggests that the 6-layered neocortex of primitive mammals evolved simultaneously from the archicortex and paleocortex. Initially, the archicortex and paleocortex respectively formed the medial and lateral sides of the cerebral hemisphere. It is likely that the 2 first gave rise to a cortex whose architecture was intermediate in complexity between the 3-layered design of the primitive cortex of origin and the "standard" 6-layered cortex. A second zone of more highly differentiated neocortex then formed as an additional concentric ring in the parahippocampal region, including the cingulate gyrus on the medial side and the insular cortex laterally. Subsequently, a third ring of well-differentiated neocortex appeared, comprising the paralimbic and parainsular cortices, which became sites of specialized sensory and motor functions. A visual cortex developed in the paralimbic zone, and part of the parainsular region became an auditory center (Table 1).

Morphologically, this marked development of the mammalian cortex occurred through an extensive infolding process, which increased its surface area significantly without a proportional enlargement of its outer dimensions or total volume. The current human cortical pattern 
is characterized by fissures, sulci, and their delimited gyral convolutions-with fissures being the most prominent and anatomically constant sulci. ${ }^{6,7}$ If one also considers the interhemispheric fissure, this invagination process led to the burial of approximately two-thirds of the human cortical surface into the depths of the sulci and fissures. ${ }^{79}$

Phylogenetically, the first hemispheric sulcus to appear was the hippocampal sulcus, which separates the archicortex (dentate gyri of the hippocampus) from its surrounding structures, including the parahippocampal subiculum, which arises during the phylogenetic and embryological caudal migration of the original supracallosal hippocampus. ${ }^{64}$ The second hemispheric sulcus was the rhinal sulcus, which demarcates the border between the paleocortex and neocortex. The rhinal sulcus appeared after the ventral displacement of the piriform cortex, caused by the development of the neocortex. ${ }^{64} \mathrm{In}$ humans, the rhinal sulcus separates the parahippocampal uncus from the rest of the neocortical temporal lobe. The hippocampal sulcus and rhinal sulcus were both already present in early mammals. ${ }^{64}$

The sylvian fissure resulted from the growth of the infolding opercula of the surrounding lobes, overlapping onto the insula, ${ }^{82}$ and became narrow only in humans, in whom the frontoparietal operculum is particularly developed (although underdeveloped in even the highest anthropoid apes). ${ }^{64,67}$ The most notable development was that of the pars triangularis and pars opercularis of the inferior frontal gyrus, which, in the dominant hemisphere of the human brain, correspond to the Broca area. ${ }^{4}$

\section{Embryological Considerations}

Embryologically, the sulci develop according to a sequence that reflects their phylogeny and a hierarchy that exists among them. Their formation begins with the appearance of the fissures, followed by sulci related to eloquent areas of the brain, and finally the secondary and tertiary cortical area sulci. ${ }^{5,11,46}$

Very early during embryogenesis, the forebrain vesicle (or prosencephalic vesicle, the most superior of the 3 primary brain vesicles originating from the neural tube) divides into the endbrain (telencephalic) and interbrain (diencephalic) vesicles. ${ }^{67}$ By approximately the 10th week of gestation, a superior midline depression of the endbrain gives rise to the interhemispheric fissure, and the transverse fissure of the brain appears between the endbrain and interbrain vesicles. ${ }^{11}$

Between the 8th and 10th weeks of gestation, transitory furrows that are not precursors of the permanent sulci appear in the cerebral hemispheric surfaces. These furrows persist until the 5th month, when the brain surfaces become smooth and the insular area is the only evident depression..$^{50,79}$

During the 4th and 5th months of gestation, the first identifiable sulci (olfactory, calcarine, parietooccipital, cingulate, and central) begin to appear, followed by additional secondary and tertiary furrows (Table 2), some of which develop only after birth. ${ }^{11,50}$

The process of sulcal development and its relatively variable final result are in great part determined by genetics. ${ }^{67}$ However, the sulci evolve through an infolding pro-
TABLE 2: Prenatal cerebral sulci development

\begin{tabular}{|c|c|c|}
\hline Characteristic & Chi et al., 1977 & Nishikuni, 2006 \\
\hline no. of fetuses & 207 & 107 \\
\hline gestational age in wks & $10-44$ & $12-40$ \\
\hline longitudinal cerebral fissure & 10 & 12 \\
\hline \multicolumn{3}{|l|}{ superolat surface } \\
\hline lat sulcus & 14 & 17 \\
\hline circular insular sulcus & 18 & 17 \\
\hline central insular sulcus & & 29 \\
\hline central sulcus & 20 & 21 \\
\hline precentral sulcus & 24 & 26 \\
\hline superior frontal sulcus & 25 & 25 \\
\hline inferior frontal sulcus & 28 & 30 \\
\hline postcentral sulcus & 25 & 29 \\
\hline intraparietal sulcus & 26 & 29 \\
\hline transverse occipital sulcus & & 30 \\
\hline lunate sulcus & & 24 \\
\hline superior temporal sulcus & 23 & 26 \\
\hline inferior temporal sulcus & 30 & 31 \\
\hline transverse temporal sulcus & 31 & 33 \\
\hline \multicolumn{3}{|l|}{ inferior surface } \\
\hline olfactory sulcus & 16 & 17 \\
\hline orbital sulcus & & 22 \\
\hline hippocampal sulcus & 10 & 12 \\
\hline rhinal sulcus & & 25 \\
\hline collateral sulcus & 23 & 29 \\
\hline occipitotemporal sulcus & 30 & 33 \\
\hline \multicolumn{3}{|l|}{ medial surface } \\
\hline callosal sulcus & 14 & 12 \\
\hline cingulate sulcus & 18 & 19 \\
\hline marginal ramus & & 33 \\
\hline paracentral sulcus & & 30 \\
\hline paraolfactory sulcus & & 29 \\
\hline subparietal sulcus & & 30 \\
\hline calcarine sulcus & 16 & 17 \\
\hline parietooccipital sulcus & 16 & 19 \\
\hline secondary sulcus & 40 & 38 \\
\hline
\end{tabular}

cess while the entire developing brain undergoes a process of circular curvature, effectively wrapping the thalami in its morphological center. Therefore, it is noteworthy that in their final presentation, the sulci of the superolateral and inferior surfaces of the cerebral hemisphere are directed toward the most proximal portion of the lateral ventricle. The development of the sulcal pattern of the medial surfaces seems to be particularly influenced by the development of the corpus callosum, since its congenital absence is linked to the absence of an arched cingulate gyrus and a radial pattern of the medial surface sulci. ${ }^{50}$

The fissures correspond to the more well-developed and anatomically constant sulci, and the gyri or convolutions that have a more rounded or quadrangular shape are usually referred to as lobules. ${ }^{5-7,27,79}$ 

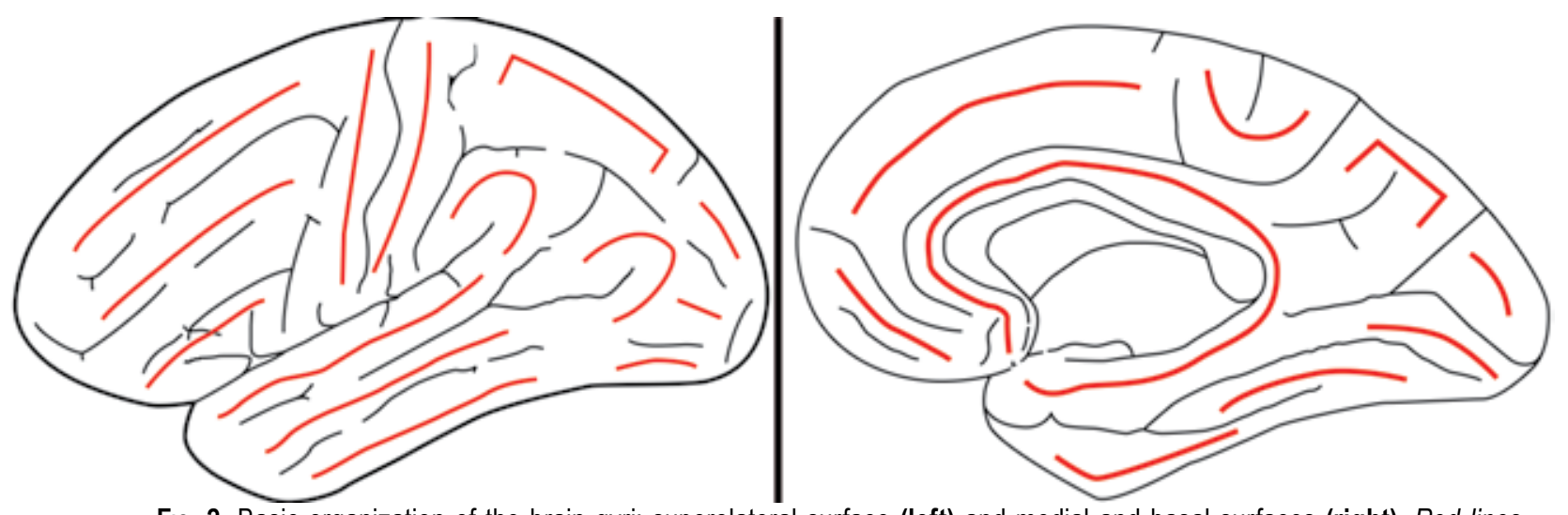

FIG. 2. Basic organization of the brain gyri: superolateral surface (left) and medial and basal surfaces (right). Red lines indicate the constant arrangement of the brain gyri.

\section{General Anatomical Features}

Given their phylogenetic and embryological development, especially the fact that it is based on an infolding process,${ }^{64}$ the fissures and sulci are natural extensions of the subarachnoid space and delimit the gyri on the brain surface, with approximate depths ranging from 1 to 3 $\mathrm{cm}$, and harbor smaller opposing gyri within their sulcal spaces. These smaller gyri are collectively known as the transverse gyri. Their wavelike surfaces are interlocked like cogwheels, and their arrangement cannot be estimated from observations of the brain surface. ${ }^{82}$ The furrows that separate the intrasulcal transverse gyri are variable in depth and length, and when they reach the superficial margins of the gyri, they are visible as incisures or notches, although furrow-like impressions can also result from indentation by arteries. ${ }^{50}$

On the brain surface, the sulci can be long or short as well as continuous (sylvian fissure, callosal, calcarine, parietooccipital, collateral, and generally the central sulcus) or interrupted. Ono et al. ${ }^{50}$ have described 4 main types of sulci: large primary sulci (for example, central, precentral, postcentral, and continuous sulci); short primary sulci (for example, rhinal, olfactory, lateral, and occipital sulci); short sulci composed of several branches (for example, orbital and subparietal sulci); and short, free supplementary sulci (for example, medial frontal and lunate sulci). Frequently, the sulci are composed of side branches that can be unconnected or connected (with end-to-side, end-to-end, or side-to-side connections that can also join 2 neighboring parallel sulci).

Since connections between sulci are common, the nomenclature varies widely, with different authors providing different interpretations. ${ }^{14,50,72}$ Bear in mind that the sulci can vary in size and shape from person to person. In addition, the cerebral gyri constitute a real continuum in that the surface presents a serpentine configuration because of the connections across the sulcal extremities and interruptions, and are continuous throughout the sulcal depths. ${ }^{82}$ The gyral separation is only superficial and is defined by the continuity and depth of the adjacent sulci. Therefore, each gyrus should be understood as a region and not as a well-defined structure.

Because they result from an infolding process, the sulci of the superolateral and inferior surfaces of the brain are usually oriented toward the nearest ventricular cavity, although this feature does not apply to the medial surface of the cerebral hemisphere, where the sulci are particularly dependent on the development of the corpus callosum. ${ }^{50}$ The single most common identifiable surface feature is the sylvian fissure, given its particular mechanism of development. ${ }^{79}$

Their variations and irregularities give the sulci and gyri of the human brain a labyrinthine appearance. Nevertheless, they are arrayed in a particular configuration. In fixed anatomical specimens from which the arachnoid and vessels have been removed, an observer with knowledge of the principal features of the sulci can generally recognize that configuration from an initial identification of the sulci that are the most characteristic and constant.

In rough terms, the human brain is organized as follows: the frontal and temporal regions of each hemisphere are each composed of 3 horizontal gyri; the central area is composed of 2 slightly oblique gyri; the parietal region is composed of 2 lobules, with a quadrangular superior lobule and an inferior lobule consisting of 2 semicircular gyri; the occipital region is composed of 3 irregular, less well-defined, predominantly longitudinal gyri that converge toward the occipital pole, the superior being vertical and the middle and inferior being horizontal; and the insula is composed of 4-5 diagonal gyri (Figs. 2 left and 3).

Medially, the external lateral gyri and lobules extend along the superior and inferolateral borders of each hemisphere. Together, these gyri constitute an outer medial ring surrounding a well-defined, C-shaped inner ring primarily composed of 2 continuous gyri. Inferiorly, the base of each hemisphere consists of 2 horizontal gyri longitudinally oriented between the lateral extended gyri (along the inferolateral and inferomedial borders) and the medial continuous gyri (of the inner ring; Figs. 2 right and 4).

Among all of these features, the prominent sylvian fissure appears as a particularly distinctive structure in the superolateral aspect of the brain, as do the uniquely oblique precentral and postcentral gyri, with their related sulci.

\section{The Cerebral Lobes}

Since the initial proposal made by Gratiolet ${ }^{6,74}$ in 

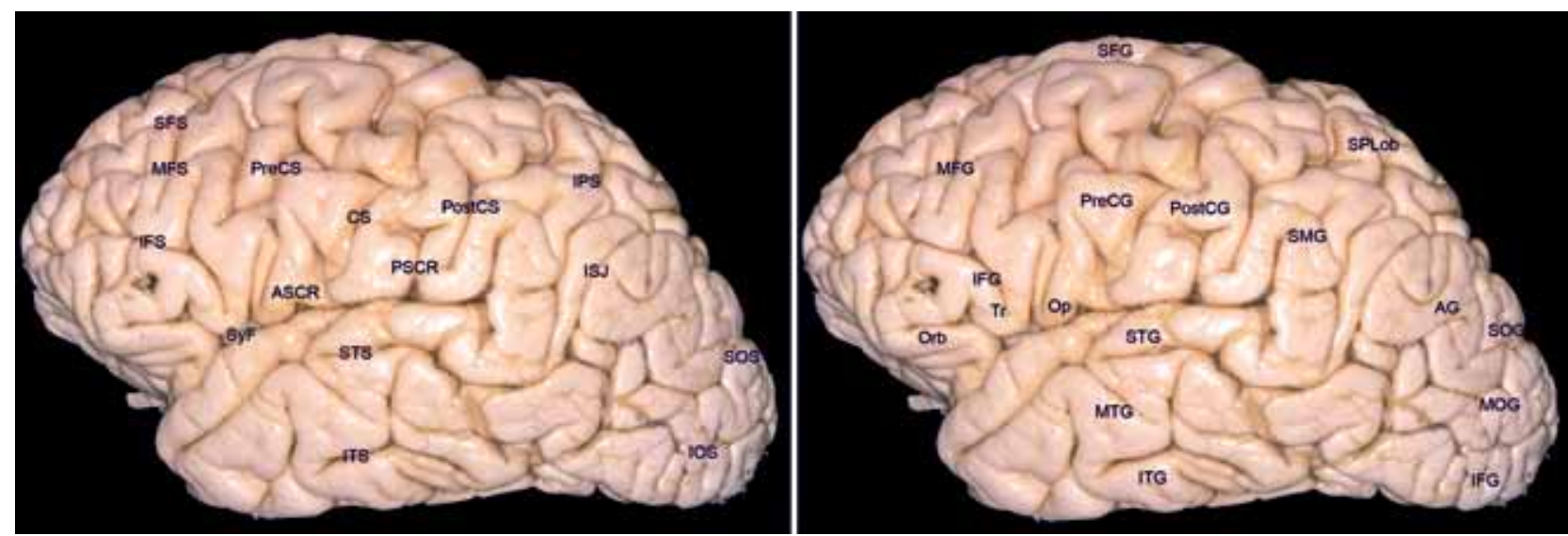

FIG. 3. The main sulci (left) and gyri (right) of the superolateral surface of the brain. $A G=$ angular gyrus; $A S C R=$ anterior subcentral ramus of sylvian fissure; CS = central sulcus; IFG = inferior frontal gyrus; IFS = inferior frontal sulcus; IOS = inferior occipital sulcus; IPS = intraparietal sulcus; ISJ = intermediary sulcus of Jensen; ITG = inferior temporal gyrus; ITS = inferior temporal sulcus; MFG = middle frontal gyrus; MFS = middle frontal sulcus; MOG = middle occipital gyrus; $M T G=$ middle temporal gyrus; Op = opercular part of inferior frontal gyrus; Orb = orbital part of inferior frontal gyrus; PostCG = postcentral gyrus; PostCS = postcentral sulcus; PreCG = precentral gyrus; PreCS = precentral sulcus; PSCR = posterior subcentral ramus of sylvian fissure; SFG = superior frontal gyrus; SFS = superior frontal sulcus; SMG = supramarginal gyrus; SOG = superior occipital gyrus; SOS = superior occipital sulcus; SPLob = superior parietal lobe; STG = superior temporal gyrus; STS = superior temporal sulcus; $\mathrm{SyF}=$ sylvian fissure; $\mathrm{Tr}=$ triangular part of inferior frontal gyrus.

the 19th century-to relate the cortical areas of the brain with the overlying skull bones previously identified by Arnold ${ }^{6,36}$ - the arbitrary division of the cerebral hemispheres into lobes has always been based particularly on anatomical aspects. The objective was to establish a system of categorization that would inform medical practice, particularly in the fields of neurology, neurosurgery, and neuroradiology.

The evolution of the official anatomical terminology, including the official description of the nomenclature related to the cerebral lobes, can be seen in successive editions of the Nomina Anatomica, which was recently superseded by the Terminologia Anatomica. ${ }^{21}$

In an initial meeting held in Basel in 1888, the International Federation of Associations of Anatomists began to systematize the anatomical nomenclature in Latin, and the first edition of the Nomina Anatomica was published in $1895 .{ }^{34}$ In this work, which came to be known as the
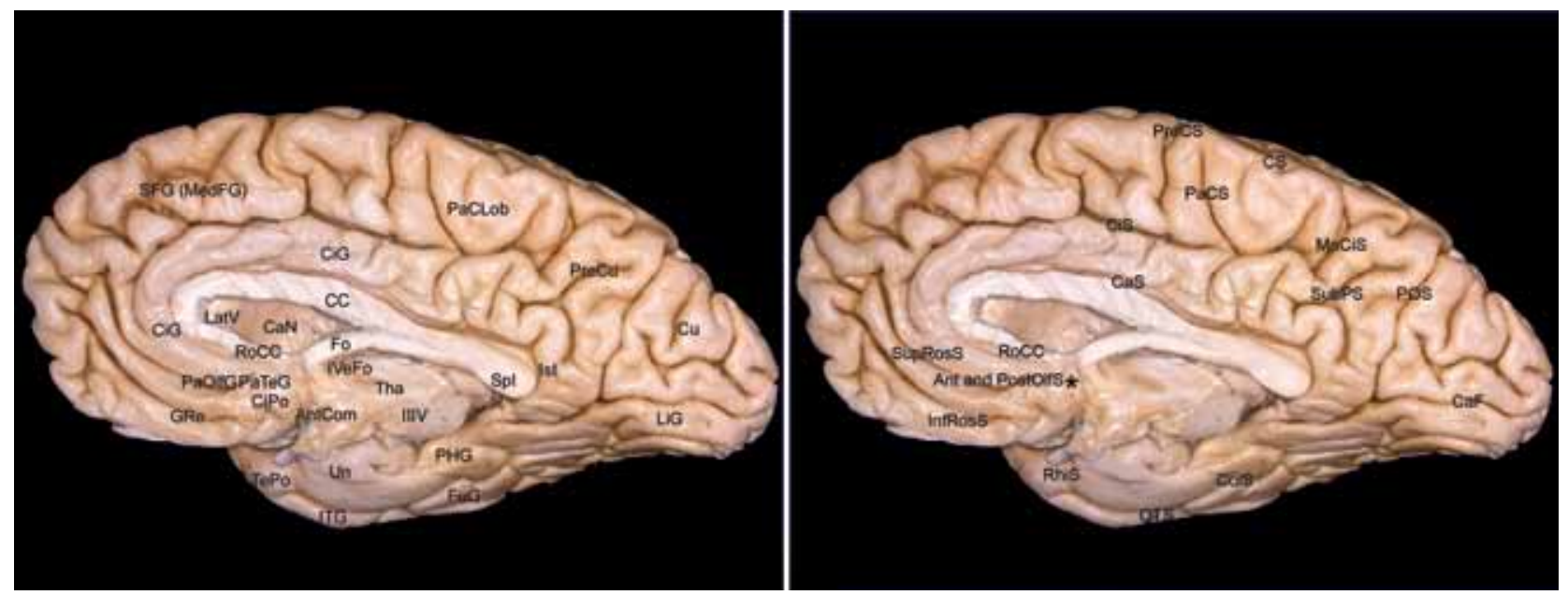

FIG. 4. The main sulci (left) and gyri (right) of the medial and basal temporooccipital surfaces. AntCom = anterior commissure; Ant and PostOlfS = anterior and posterior paraolfactory sulcus; $\mathrm{CaF}=$ calcarine fissure; $\mathrm{CaN}=$ caudate nucleus; CaS = callosal sulcus; $\mathrm{CC}=$ corpus callosum; $\mathrm{CiG}=$ cingulate gyrus; $\mathrm{CiPo}=$ cingulate pole; $\mathrm{CiS}=$ cingulate sulcus; $\mathrm{ColS}=$ collateral sulcus; $\mathrm{CS}$ = central sulcus; $\mathrm{Cu}$ = cuneus; Fo = fornix; FuG = fusiform gyrus; GRe = gyrus rectus; IIIV = third ventricle; InfRosS = inferior rostral sulcus; Ist = isthmus of cingulate gyrus; ITG = inferior temporal gyrus; IVeFo = interventricular foramen of Monro; LatV = lateral ventricle; LiG = lingual gyrus; MaCiS = marginal ramus of the cingulate sulcus; MedFG = medial frontal gyrus; OTS = occipitotemporal sulcus; PaCLob = paracentral lobule; $\mathrm{PaCS}=$ paracentral sulcus; $\mathrm{PaOlfG}=$ paraolfactory gyri; $\mathrm{PaTeG}=$ paraterminal gyrus; $P H G$ = parahippocampal gyri; $P O S$ = parietooccipital sulcus; PreCS = precentral sulcus; PreCu = precuneus; RhiS = rhinal sulcus; RoCC = rostrum of the corpus callosum; SFG = superior frontal gyrus; Spl = splenium of corpus callosum; SubPS = subparietal sulcus; SupRosS = superior rostral sulcus; TePo = temporal pole; Tha = thalamus; Un = uncus. 
Basle Nomina Anatomica, each hemisphere of the brain was divided into frontal, parietal, occipital, and temporal lobes, and the insula was considered a separate addendum but not a lobe.

The second edition of the Nomina Anatomica, published in 1935, became known as the Jena Nomina Anatomica ${ }^{68}$ and maintained the same cerebral subdivisions, as did subsequent quinquennial editions. In 1955 the $\mathrm{Pa}$ risiensia Nomina Anatomica ${ }^{20}$ was published. That edition also presented no alterations in the terms of the cerebral subdivisions. The Nomina Anatomica then came to be considered an international anatomical reference.

Just after the Tenth International Congress of Anatomists, held in Tokyo in 1975, the fourth edition of the original Parisiensia Nomina Anatomica was published..$^{18}$ In that edition, the insula was designated a brain lobe. The fifth edition, ${ }^{19}$ approved at a subsequent meeting held in Mexico City in 1980, presented no further modifications regarding the cerebral lobes.

At the Federative World Congress of Anatomy, held in Rio de Janeiro in 1989, a Federative Committee on Anatomical Terminology (FCAT) was elected. In several meetings over the next 8 years, the committee "reviewed and revised the anatomical Latin terminology with the addition of a list of English terms in common usage" and published the Terminologia Anatomica in 1998.21 The Terminologia Anatomica contains the contemporary official international anatomical terminology and designates the limbic lobe as another cerebral subdivision. Therefore, according to the current official anatomical nomenclature, the brain is divided into 6 lobes: frontal, parietal, occipital, temporal, insular, and limbic.

The development of and advances in microneurosurgery during the latter part of the 20th century, guided in particular by the contributions of Yaşargil, ${ }^{80}$ led initially to the transcisternal approaches, ${ }^{81,85}$ which gave rise to the transfissural (for extrinsic lesions) and transsulcal approach (for intrinsic lesions). ${ }^{28,52,82,83,84}$ The consequent microsurgical possibilities naturally required anatomical and topographic descriptions that were more accurate and detailed. In 1990 Ono et al. ${ }^{50}$ studied the details of the main cerebral sulci. More recently, Yaşargil provided a complete and didactic description of the cerebral sulci and gyri, dividing each cerebral hemisphere into 7 lobes-frontal, central, parietal, occipital, temporal, insular, and limbic ${ }^{82}$-and that division is adopted in the present article. The concept of a central lobe composed of the precentral and postcentral gyri is justified, since they compose a single morphological and functional unit, as has been suggested by Penfield and Rasmussen ${ }^{51}$ and Rasmussen alone. ${ }^{54-56}$

\section{The Sulci, Gyri, and Cerebral Lobes}

Given the visual evidence of the lateral cerebral sulcus, or sylvian fissure, together with the distinct, slightly oblique arrangement of the precentral and postcentral gyri as well as of the sulci that delineate them at approximately the center of the external brain surface, the character of the remaining gyri of the superolateral surface of each cerebral hemisphere can be more easily understood if we take as our starting point and basis those sulcal spaces and cerebral gyri. The macroscopic study of the sulci and gyri of each cerebral hemisphere should therefore begin with the identification of the sylvian fissure, which clearly separates the superolateral surfaces of the frontal, central, and parietal lobes from the temporal lobe, and should be followed by the identification of the precentral and postcentral gyri, which divide the portion of this surface that is superior and posterior to the sylvian fissure into its anterior and posterior halves. Adopting this approach, we will describe the 7 lobes of each cerebral hemisphere as follows: frontal, central, parietal, occipital, temporal, insular, and limbic. ${ }^{82}$ Since the connections of the limbic lobe are particularly complex, the text related to that lobe also addresses the adjacent and correlated areas.

\section{The Frontal Lobe}

The frontal lobe constitutes the largest, most anterior part of each hemisphere. Within the scheme adopted in this article, the frontal lobe is delineated posteriorly by the oblique precentral sulcus and is composed of the superior, middle, and inferior frontal gyri, which are oriented longitudinally and separated by the superior and inferior frontal sulci, also longitudinally oriented (Fig. 3). These gyri are often referred to as F1, F2, and F3, respectively.

On the cerebral surface posteriorly, the superior frontal gyrus is connected to the precentral gyrus by at least 1 fold, which more commonly lies medially along the interhemispheric fissure. Anteriorly, the superior frontal gyrus might be connected to the middle frontal gyrus, with the orbital gyri and the gyrus rectus. In general terms, the superior frontal gyrus is subdivided into 2 longitudinal portions by the so-called medial frontal sulcus, and its medial portion is at times designated the medial frontal gyrus. ${ }^{50}$ Along the most medial portion of the superior frontal gyrus and immediately facing the precentral gyrus is the important region known as the supplementary motor area, which varies from person to person and has poorly defined borders..$^{8,79}$

The middle frontal gyrus is typically the largest of the frontal gyri and often has a sulcus that is shallower than that of the other frontal gyri, usually running along its anterior two-thirds and known as the middle or intermediate frontal sulcus. ${ }^{50}$ In most human brains, the middle frontal gyrus is superficially connected to the precentral gyrus by a prominent root that lies between the extremities of a marked interruption in the precentral sulcus. The frequent interruptions in the inferior frontal sulcus are attributable to connections between the middle and inferior frontal gyri.

The inferior frontal gyrus is irregular, crisscrossed by various small branches of the inferior frontal sulcus, which typically superiorly delineate the gyrus in an interrupted manner. Inferiorly, this gyrus is delineated and crisscrossed by rami of the sylvian fissure. Anteriorly, the inferior frontal gyrus terminates, merging with the anterior portion of the middle frontal gyrus. Posteriorly, it is connected to the precentral gyrus. The inferior frontal gyrus is composed of, from anterior to posterior, its orbital, triangular, and opercular parts.

The emergence of the horizontal and ascending ante- 

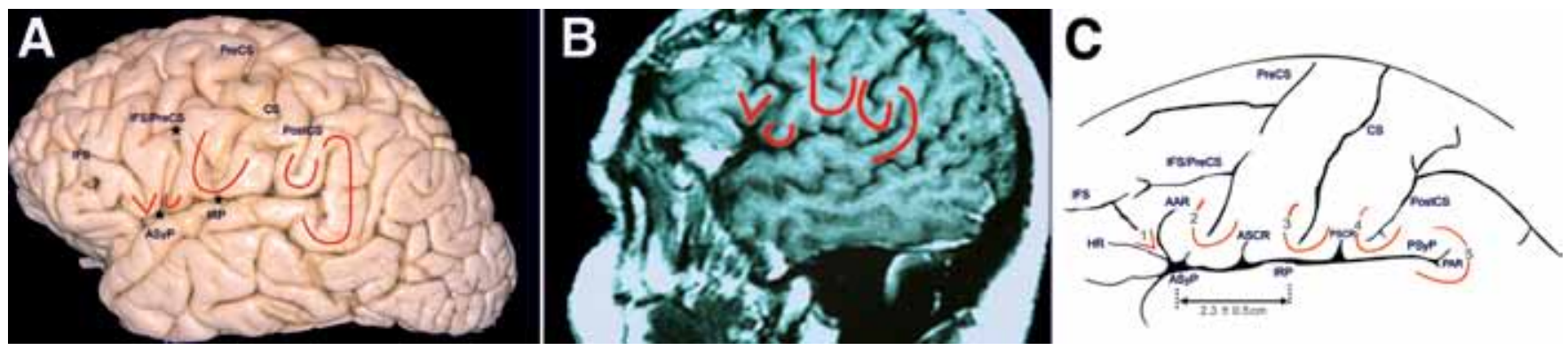

FIG. 5. The frontoparietal operculum: cadaveric specimen (A), MR image (B), and sketch of sulcal and gyri morphology (C). The frontoparietal operculum is characterized by a V-shaped convolution consisting of the triangular part of the inferior frontal gyrus (IFG) (1), located just superiorly to the anterior sylvian point (ASyP), and usually containing a descending branch of the inferior frontal sulcus (IFS); the 3 following U-shaped convolutions respectively composed by the opercular part of the IFG (2), which is always intersected by the inferior part of the precentral sulcus; the subcentral gyrus or rolandic operculum (3), composed of the inferior connection of the pre- and postcentral gyri enclosing the inferior part of the central sulcus (CS); the connection arm between the postcentral and supramarginal gyri (4) that contains the inferior part of the postcentral sulcus; and finally the Cshaped convolution (5) constituted by the connection of the supramarginal and superior temporal gyri that encircles the posterior end of the sylvian fissure. The bottoms of the $U$-shaped convolutions and their related sulcal extremities can be situated either superior to the fissure or inside its cleft. Stars designate the areas labeled. AAR = anterior ascending ramus of the sylvian fissure; ASCR = anterior subcentral ramus of sylvian fissure; HR = horizontal ramus of sylvian fissure; IFS/PreCS = IFS and precentral sulcus; IRP = inferior rolandic point, projection of the central sulcus in the sylvan fissure; PAR = posterior ascending ramus of sylvian fissure; PostCS = postcentral sulcus; PreCS = precentral sulcus; PSCR = posterior subcentral ramus of sylvian fissure; PSyP = posterior sylvian point.

rior rami from the same point in the sylvian fissure characterizes the triangular part of the inferior frontal gyrus, which is typically more retracted than the other 2 parts (Figs. 5 and 6). The orbital part is the most prominent of the 3 , and the opercular part is consistently U-shaped. Given the usual retraction of the triangular part, the horizontal and ascending anterior branches of the sylvian fissure typically emerge from a small widening of the subarachnoid space, designated the anterior sylvian point. ${ }^{61,86}$ Therefore, the anterior sylvian point is situated inferior to the triangular part and anterior to the base of the opercular portion. The point, which is typically visible to the naked eye, divides the sylvian fissure into its anterior and posterior branches. ${ }^{61,86}$
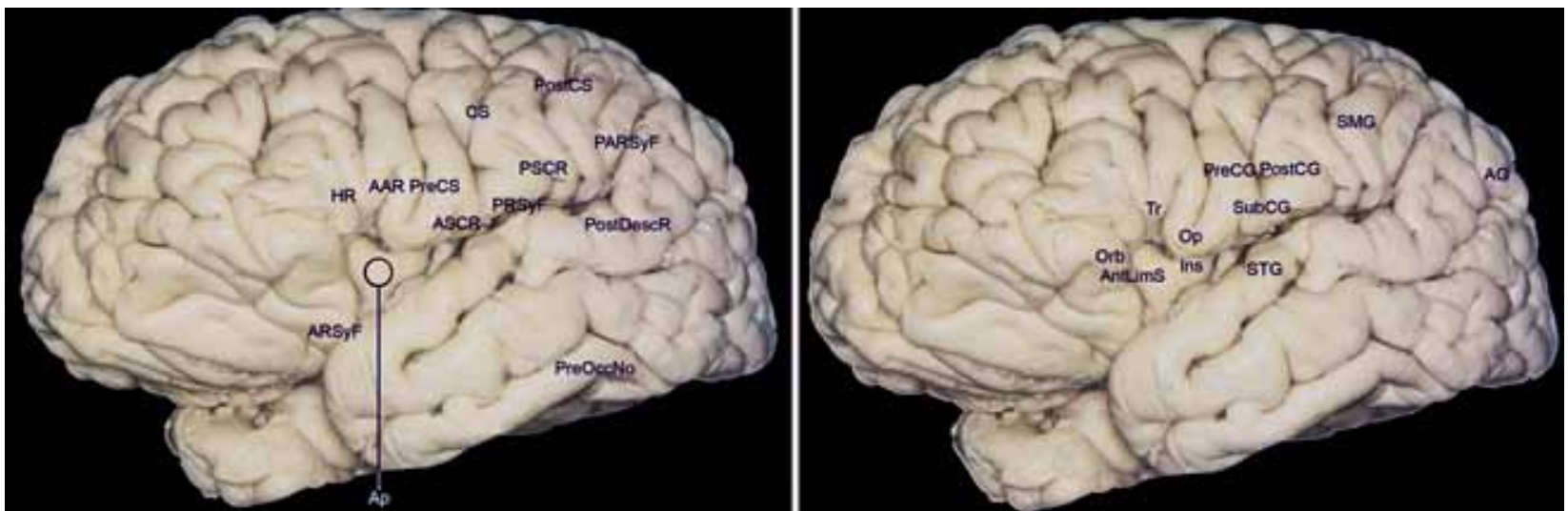

Fig. 6. Sulci (left) and gyri (right) of the frontoorbital, frontoparietal, and temporal operculi. $A A R=$ anterior ascending ramus of the sylvian fissure; $A G$ = angular gyrus; $A$ ntLimS = anterior limiting sulcus of the insula; $A p=$ insular apex; $A R S y F=$ anterior ramus of sylvian fissure; $A S C R$ = anterior subcentral ramus of sylvian fissure; $C S=$ central sulcus; $H R=$ horizontal ramus of sylvian fissure; Ins = insula; Op = opercular part of inferior frontal gyrus; Orb = orbital part of inferior frongal gyrus; PARSyF = posterior ramus of sylvian fissure; PostCG = postcentral gyrus; PostCS = postcentral sulcus; PostDescR = posterior descending ramus of sylvian fissure; PreCG = precentral gyrus; PreCS = precentral sulcus; PreOccNo = preoccipital notch; PRSyF = posterior ramus of sylvian fissure; PSCR = posterior subcentral ramus of sylvian fissure; SMG = supramarginal gyrus; $S T G$ = superior temporal gyrus; SubCG = subcentral gyrus; $\mathrm{Tr}=$ triangular part of inferior frontal gyrus. 


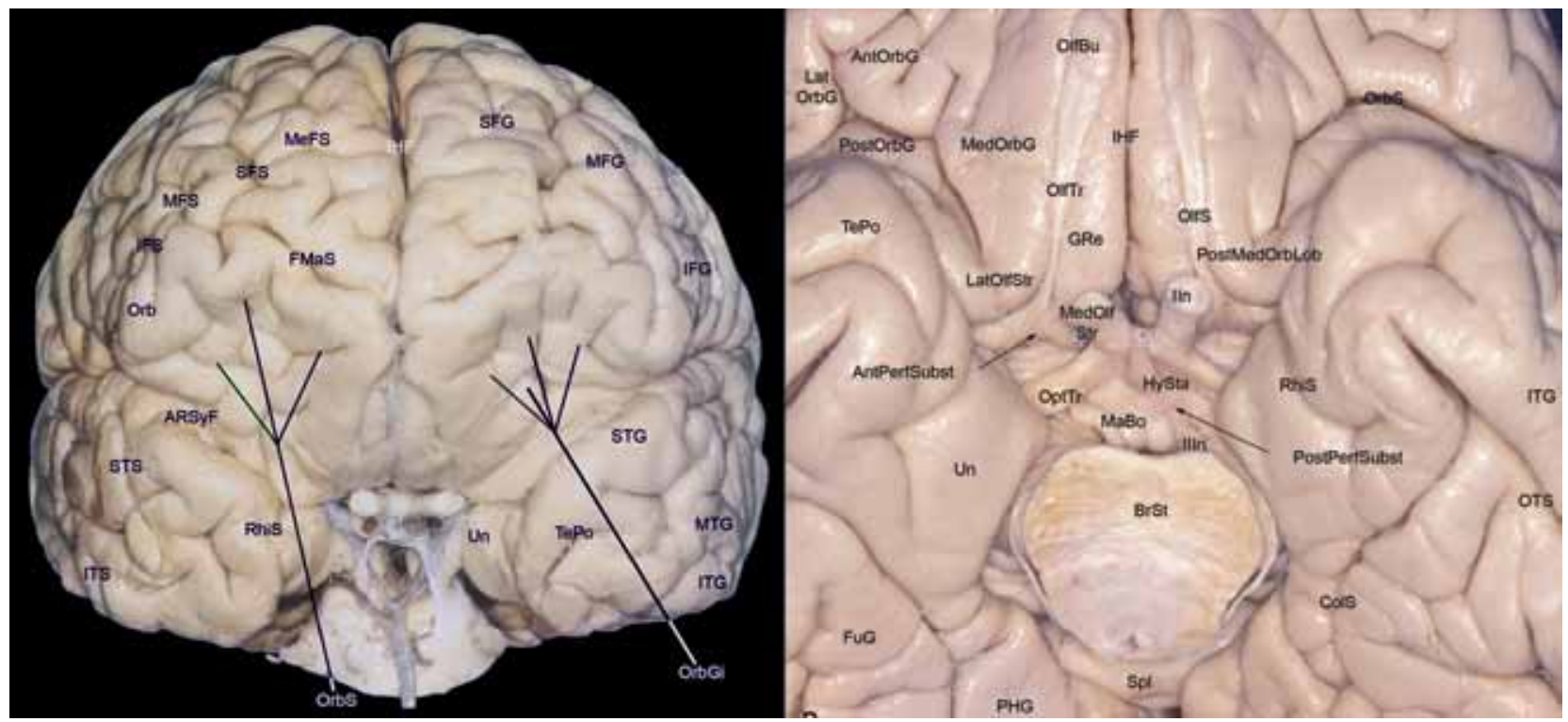

FIG. 7. Anterior view of cerebral hemispheres (left) and view of the basal frontotemporal surface (right). AntOrbG = anterior orbital gyrus; AntPerfSubst = anterior perforated substance; $\mathrm{ARSyF}=$ anterior ramus of sylvian fissure; $\mathrm{BrSt}=$ brainstem (pons); $\mathrm{ColS}=$ collateral sulcus; FMaS = frontomarginal sulcus; FuG = fusiform gyrus; GRe = gyrus rectus; HySta = hypophyseal stalk; IFG = inferior frontal gyrus; IFS = inferior frontal sulcus; IHF = interhemispheric fissure; Ist = isthmus of cingulate gyrus; ITG = inferior temporal gyrus; ITS = inferior temporal sulcus; LatOlfStr = lateral olfactory striae; LatOrbG = lateral orbital gyrus; MaBo = mamillary body; MedOlfStr = medial olfactory striae; MedOrbG = medial orbital gyrus; MeFS = medial frontal sulcus; $M F G$ = middle frontal gyrus; MFS = middle frontal sulcus; MTG = middle temporal gyrus; OlfBu = olfactory bulb; OlfS = olfactory sulcus; OlfTr = olfactory tract; OptTr = optic tract; Orb = orbital part of inferior frontal gyrus; OrbGi = orbital gyri; OrbS = orbital sulcus; OTS = occipitotemporal sulcus; PHG = parahippocampal gyri; PostMedOrbLob = posteromedial orbital lobule; PostOrbG = posterior orbital gyrus; PostPerfSubst = posterior perforated substance; RhiS = rhinal sulcus; SFG = superior frontal gyrus; SFS = superior frontal sulcus; Spl = splenium of corpus callosum; STG = superior temporal gyrus; STS = superior temporal sulcus; TePo = temporal pole; Un = uncus; IIn = optical nerve; IIIn = oculomotor nerve.

Eberstaller. When the diagonal sulcus of Eberstaller is present, it divides the anterior portion of the opercular part into 2 triangular portions that are positioned inversely to each other.

In the dominant hemisphere, the opercular and triangular parts of the inferior gyrus correspond to the Broca area, which is responsible for the production of spoken language. . $^{4,29,53,79}$

Inferiorly, although the orbital part continues with the lateral orbital gyrus, at times passing under a shallow sulcus known as the frontoorbital sulcus, the triangular part is always superior to the sylvian fissure, and the base of the opercular part can be located either superiorly or within that same fissure. ${ }^{50,60}$

The triangular and opercular parts together with the subcentral gyrus, which connects the precentral and postcentral gyri, and the anteroinferior portion of the supramarginal gyrus cover the superior aspect of the insular surface and constitute the frontoparietal operculum (Figs. 5 and 6). Therefore, the frontoparietal operculum is situated between the horizontal and posterior ascending branches of the sylvian fissure..$^{61,79}$

Anteriorly, the frontal gyri are delineated by the appropriately named frontomarginal sulcus, which lies superior and parallel to the supraciliary margin, separating the superolateral and orbital frontal surfaces..$^{50,82}$

On the frontobasal, or orbital, surface of each frontal lobe, the deep olfactory sulcus, which harbors the olfac- tory bulb and olfactory tract, is very deep and lies longitudinally in a paramedian position (Fig. 7). Posteriorly, the olfactory tract is divided into the medial and lateral striae, which delineate the anteriormost aspect of the anterior perforated substance.

Medial to the olfactory sulcus is the long and narrow gyrus rectus, considered the most anatomically constant of the cerebral gyri, which is continuous with the superior frontal gyrus along the medial surface of the hemisphere (Fig. 4).

Lateral to the olfactory sulcus are the orbital gyri, which account for the greatest proportion of the frontobasal surface (Fig. 7). The $\mathrm{H}$-shaped orbital sulcus (cruciform sulcus of Rolando) delineates the anterior, posterior, medial, and lateral orbital gyri. The posterior orbital gyrus is situated anterior to the anterior perforated substance and typically presents a configuration similar to a tricorner or "Napoleon" hat, which can facilitate its identification in anatomical specimens in which the $\mathrm{H}$-shaped orbital sulcus presents variations.

The posterior orbital gyrus is connected medially to the medial orbital gyrus, characterizing the posteromedial orbital lobule situated posterior and along the olfactory tract and the lateral olfactory striae, which is in turn connected to the anterior portion of the insula via the transverse insular gyrus. The remaining orbital gyri are connected to the superior, middle, and inferior frontal gyri, along the frontal pole. 


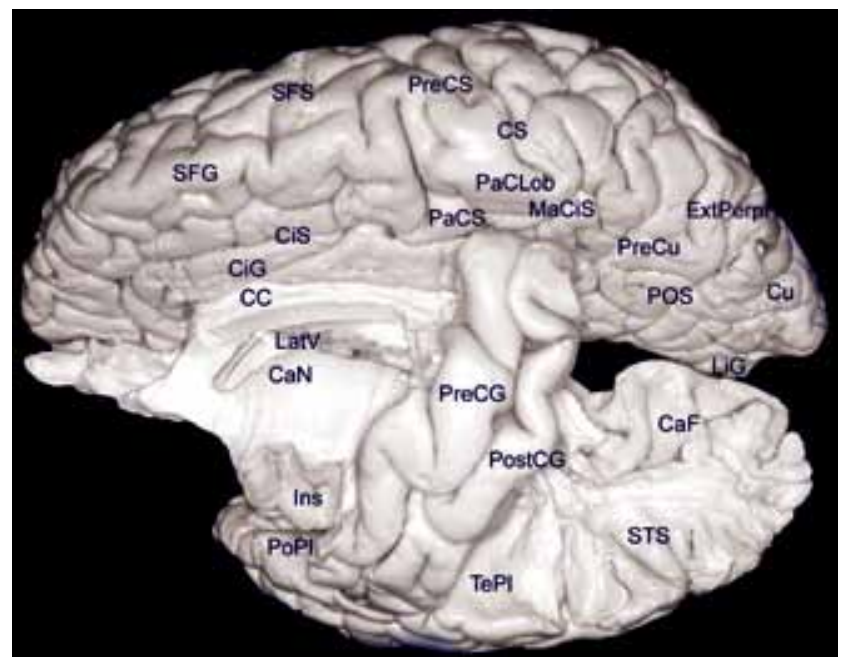

FIG. 8. The central lobe and medial contralateral surface. $\mathrm{CaF}=$ calcarine fissure; $\mathrm{CaN}=$ caudate nucleus; $\mathrm{CC}=$ corpus callosum; $\mathrm{CiG}$ = cingulate gyrus; $\mathrm{CiS}=$ cingulate sulcus; $\mathrm{CS}=$ central sulcus; $\mathrm{Cu}=$ cuneus; ExtPerpF = external perpendicular fissure; Ins = insula; LatV = lateral ventricle; $\mathrm{LiG}=$ lingual gyrus; $\mathrm{MaCiS}=$ marginal ramus of the cingulate sulcus; $\mathrm{PaCLob}=$ paracentral lobule; $\mathrm{PaCS}=$ paracentral sulcus; $\mathrm{PoPI}=$ polar plane of the opercular temporal surface; $\mathrm{POS}$ = parietooccipital sulcus; PostCG $=$ postcentral gyrus; PreCG $=$ pre central gyrus; PreCS = precentral sulcus; $\mathrm{PreCu}=$ precuneus; $\mathrm{SFG}=$ superior frontal gyrus; SFS = superior frontal sulcus; STS = superior temporal sulcus; TePI = temporal plane.

\section{The Central Lobe}

The central lobe consists of the precentral (motor gyrus) and postcentral gyri (sensitive gyrus; Fig. 8), which are oriented obliquely on the superolateral surface and are separated by the central sulcus, by their inferior (subcentral gyrus) and superior connections (paracentral gyrus, or lobule, located on the medial surface) and by the other related sulci. ${ }^{82}$

Morphologically, the central lobe is situated obliquely over the sylvian fissure corresponding approximately to the median portion of the cerebral hemisphere.

On the superolateral surface, the central lobe is delineated anteriorly by the precentral and anterior subcentral sulci, and posteriorly by the postcentral and posterior subcentral sulci (Figs. 3, 5, and 6). On the medial surface of the cerebral hemisphere (Fig. 4), the paracentral lobule is delineated anteriorly by the paracentral sulcus, and inferiorly and posteriorly by the ascending and distal part of the cingulate sulcus, which is known as the marginal ramus of the cingulate sulcus. ${ }^{50}$

The precentral and postcentral gyri are situated obliquely in relation to the interhemispheric fissure, being less serpiginous than the other gyri of the cerebral convexity, and are connected to adjacent gyri via the usual interruptions in the precentral and postcentral sulci.

The precentral and postcentral gyri are consistently united inferiorly by the subcentral gyrus (Broca's inferior frontoparietal pli de passage, or rolandic operculum) and superiorly by the paracentral lobule (Broca's superior frontoparietal pli de passage), which is located on the medial surface of each hemisphere. The precentral and postcentral gyri together resemble an elongated ellipse that is furrowed by the central sulcus, which is usually continuous, and are respectively delineated anteriorly and posteriorly by the precentral and postcentral sulci, which are typically discontinuous. This morphological unit, together with the functional interaction between motricity and sensitivity, justifies the characterization of these gyri as constituting a single lobe.

Inferiorly, the subcentral gyrus is delineated anteriorly and posteriorly by the anterior and posterior subcentral rami of the sylvian fissure, respectively. It can be situated either completely over the sylvian fissure or in part internal to the fissure, giving the false impression that the central sulcus is a branch of the sylvian fissure..$^{60,61}$ The portion of the subcentral gyrus that corresponds to the base of the postcentral gyrus consistently lies over the transverse gyrus of Heschl, which is situated on the opercular surface of the temporal lobe. ${ }^{78}$

Superiorly, and situated in the interhemispheric fissure, the paracentral lobule is delineated anteriorly by the paracentral sulcus and posteriorly by distal part of the cingulate sulcus, that is, the ascending marginal ramus of the cingulate sulcus.

The precentral gyrus typically presents 3 prominences known as knees: the superior and inferior knees are characterized by anterior convexities, and the middle knee is characterized by a posterior convexity. The precentral gyrus, in addition to its superior and inferior connections with the postcentral gyrus via the superior (paracentral lobule) and inferior frontoparietal fold (subcentral gyrus, or rolandic operculum), is usually also connected to the postcentral gyrus via a transverse gyrus that lies along the bottom of the central sulcus and constitutes the so-called Broca's medial frontoparietal pli de passage.,72 This fold is situated at the level of the middle knee of the precentral gyrus, which is itself normally situated at the level of the superior frontal sulcus, corresponding to the portion of the gyrus that functionally harbors the motor representation of the contralateral hand. Therefore, the superior frontal sulcus tends to point the way to the medial frontoparietal pli de passage, as well as to the middle knee of the precentral gyrus, with its respective motor representation of the hand. ${ }^{2}$ On axial MR images, this part of the precentral gyrus frequently presents a configuration that resembles the Greek letter $\omega .^{2}$

Since they are disposed obliquely, the superior portions of the precentral and postcentral gyri that constitute the paracentral lobule on the medial surface of the cerebral hemisphere are topographically related to the ventricular atrium, which is situated posteriorly to the thalamus (Fig. 8). In contrast, the inferior portions cover the posterior half of the insula and are topographically related to the body of the lateral ventricle, which is situated superior to the thalamus.

\section{The Parietal Lobe}

The parietal lobe anatomy is more complex in the sense that it is composed of gyri structurally less well defined and particularly serpiginous and curved. These gyri are also referred to as lobules.

On the superolateral surface, the parietal lobe is de- 


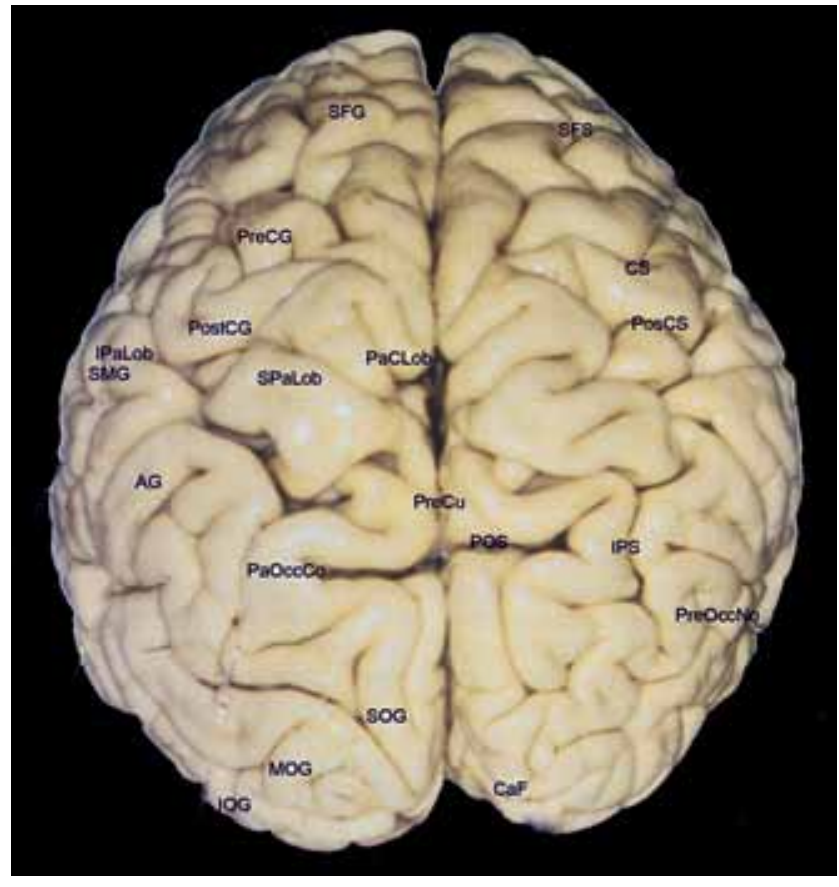

FIG. 9. Superior view of the cerebral hemispheres. $A G=$ angular gyrus; $\mathrm{CaF}=$ calcarine fissure; $\mathrm{CS}=$ central sulcus; $\mathrm{IOG}=$ inferior occipital gyrus; IPaLob = inferior parietal lobule; IPS = intraparietal sulcus; $M O G=$ middle occipital gyrus; $P a C L o b=$ paracentral lobule; $\mathrm{PaOccCo}=$ parietooccipital arch; $\mathrm{POS}=$ parietooccipital sulcus; PosCS = postcentral sulcus; PostCG = postcentral gyrus; PreCG = precentral gyrus; PreCu = precuneus; PreOccNo = preoccipital notch; SFG = superior frontal gyrus; $S F S=$ superior frontal sulcus; $S M G=$ supramarginal gyrus; SOG = superior occipital gyrus; SPaLob = superior parietal lobe.

lineated anteriorly by the postcentral sulcus and posteriorly by the imaginary line running from the point from which the parietooccipital sulcus emerges (on the superomedial border) to the preoccipital notch, which is situated on the inferolateral border, $\sim 5 \mathrm{~cm}$ anterior to the occipital pole..$^{79}$ The intraparietal sulcus, which originates from around the midpoint of the postcentral sulcus, is prominent along the parietal superolateral surface, in general runs almost parallel to the interhemispheric fissure, and posteriorly penetrates into the occipital lobe. The intraparietal sulcus divides the superolateral parietal surface into the superior and inferior parietal lobules (Fig. 9).

The superior parietal lobule is quadrangular and typically connected to the postcentral gyrus via a connection that transects the most superior portion of the postcentral sulcus and, occasionally, via another fold that more inferiorly interrupts the postcentral sulcus. The superior parietal lobule is delineated laterally by the intraparietal sulcus; medially, it is continuous with the precuneus gyrus along the superomedial border (Fig. 4); and, posteriorly, it continues to the superior occipital gyrus via the prominent and arched superior parietooccipital fold that surrounds the external perpndicular fissure, which represents the depth of the parietooccipital sulcus over the superolateral cerebral surface (Fig. 9). In some brains, there is also a small sulcus, designated the superior parietal sulcus, emerging from the interhemispheric fissure, between the postcentral sulcus and the external perpendicular fissure, over the superior parietal lobule. ${ }^{50}$

The inferior parietal lobule consists of, anteriorly, the supramarginal gyrus, which is a curved gyrus surrounding the distal portion of the sylvian fissure, and, posteriorly, the angular gyrus, which encircles the horizontal distal portion of the superior temporal sulcus. The supramarginal and angular gyri characterize the cranial parietal tuberosity, or bossa. Those 2 gyri are separated by the intermediate sulcus ${ }^{77}$ (of Jensen), which is an inferior vertical branch of the intraparietal sulcus or a distal and superior vertical branch of the superior temporal sulcus or both (Fig. 3).

Anteriorly, the supramarginal gyrus is connected to the postcentral gyrus via a fold that lies around the inferior portion of the postcentral sulcus; inferiorly, it consistently encircles the terminal portion of the sylvian fissure and continues to the superior temporal gyrus; and posteriorly, it occasionally rounds the inferior border of the intermediate sulcus, connecting to the angular gyrus (Figs. 3 and 5). In turn, the angular gyrus typically curves anteriorly around a distal horizontal branch of the superior temporal sulcus, also known as the angular sulcus ${ }^{50}$ continuing to the middle temporal gyrus and giving rise to a posterior fold that connects it to the middle occipital gyrus. (Broca, basing his ideas on the work of Gratiolet, considered the supramarginal and angular gyri to be folds connecting the parietal lobe with the temporal lobe. From Broca's perspective, the supramarginal gyrus, which wraps around the distal portion of the sylvian fissure, corresponded to Gratiolet's superior marginal fold, and the angular gyrus, which wraps around the distal portion of the superior temporal sulcus, corresponded to the inferior marginal fold, or curved fold. ${ }^{71,72}$ )

Therefore, the intraparietal sulcus delineates superiorly the supramarginal and angular gyri with a slightly arciform and inferiorly concave course, and, anteriorly, it typically continues to the inferior portion of the postcentral sulcus. Posteriorly, the continuation of the intraparietal sulcus becomes the intraoccipital sulcus, ${ }^{14,44}$ also known as the superior occipital sulcus ${ }^{71}$ or transverse occipital sulcus, ${ }^{50}$ which separates the (more vertical) superior occipital gyrus from its (more horizontal) middle counterpart. ${ }^{14,50,71,72}$ Along its length, the intraparietal sulcus typically gives rise to 2 vertical folds: a smaller, superior fold located anterior to the external perpendicular fissure, known as the transverse parietal sulcus of Brissaud, and another inferior, more developed fold that constitutes the previously mentioned intermediate sulcus of Jensen, which separates the supramarginal gyrus from the angular gyrus. ${ }^{71,72,77}$ The superior parietal lobule, supramarginal gyrus, and angular gyrus are also known as $\mathrm{P} 1, \mathrm{P} 2$, and $\mathrm{P} 3$, respectively.

On the medial surface of each hemisphere, the precuneus gyrus is a medial extension of the superior parietal lobule along the superomedial border of the brain and corresponds to to the medial portion of the parietal lobe (Fig. 4). The precuneus is also quadrangular, delineated anteriorly by the marginal branch of the cingulate sulcus, posteriorly by the parietooccipital sulcus, and inferiorly by the subparietal sulcus. Inferiorly to the subparietal 
sulcus, the precuneus is connected to the isthmus of the cingulate gyrus and the parahippocampal gyrus.

\section{The Occipital Lobe}

On the superolateral cerebral surface, the occipital lobe is situated posterior to the imaginary line that connects the point of emergence of the parietooccipital sulcus (on the superomedial border of the cerebral hemisphere) with the preoccipital notch. The sulci and gyri of the occipital lobe have greater anatomical variation as compared with other lobes. Despite being less well defined and less anatomically constant than gyri in other dorsal cortical areas, the occipital gyri of the superolateral cerebral surface tend to consist of 3 gyri that are, for the most part, arrayed longitudinally in relation to the interhemispheric fissure and converge posteriorly to form the occipital pole of each hemisphere. As is the case for the frontal and temporal lobes, the occipital gyri of the superolateral surface are usually designated superior, middle, and inferior, ${ }^{14,71}$ or $\mathrm{O} 1, \mathrm{O} 2$, and $\mathrm{O} 3$, respectively. While the superior occipital gyrus is arranged more vertically along the interhemispheric fissure, the middle and inferior occipital gyri are arranged more horizontally and parallel to the inferior cerebral margin (Fig. 9).

On the medial surface, the occipital lobe is particularly well defined anatomically. It is separated from the parietal lobe by the parietooccipital sulcus and is composed of the cuneal and lingual gyri, which are separated by the calcarine fissure (Fig. 4). The basal, or inferior, surface of the occipital lobe, in turn, is contiguous with the basal surface of the temporal lobe (Fig. 10).

On the superolateral surface, whereas the superior and middle occipital gyri are separated by the intraoccipital sulcus, ${ }^{14,44,71}$ which is the continuation of the intraparietal sulcus and is also known as the superior occipital sulcus ${ }^{14,71}$ and transverse occipital sulcus, ${ }^{50}$ the middle and inferior occipital gyri are separated by the less welldefined inferior occipital sulcus, ${ }^{14,71}$ also known as the lateral occipital sulcus. ${ }^{50}$ When present, the so-called lunate sulcus is typically oriented vertically, immediately facing the occipital pole. ${ }^{14,50}$ Given the shallow depth and discontinuity as well as the (often) multiple branches of the 2 principal occipital sulci, the occipital gyri are not always well defined and are typically joined by various anastomotic folds, and thus constitute a cortical surface that is difficult to characterize morphologically. (According to the description given by Gratiolet, the parietal and temporal lobe connections with the occipital lobe are made via 4 folds [2 parietooccipital and 2 temporooccipital]: 1 ) the first and more superior parietooccipital fold, which surrounds the external perpendicular occipital fissure and connects the superior parietal lobule with the superior occipital gyrus; 2) the second and more inferior parietooccipital fold, composed of a posterior extension of the angular gyrus, which converges with the middle occipital gyrus and also occasionally with the superior occipital gyrus; 3 ) the first temporooccipital fold, characterized by the union of the middle temporal gyrus and the inferior occipital gyrus; and 4) the second temporooccipital fold, characterized by the union of the inferior temporal gyrus and the inferior occipital gyrus. ${ }^{71,72}$ )

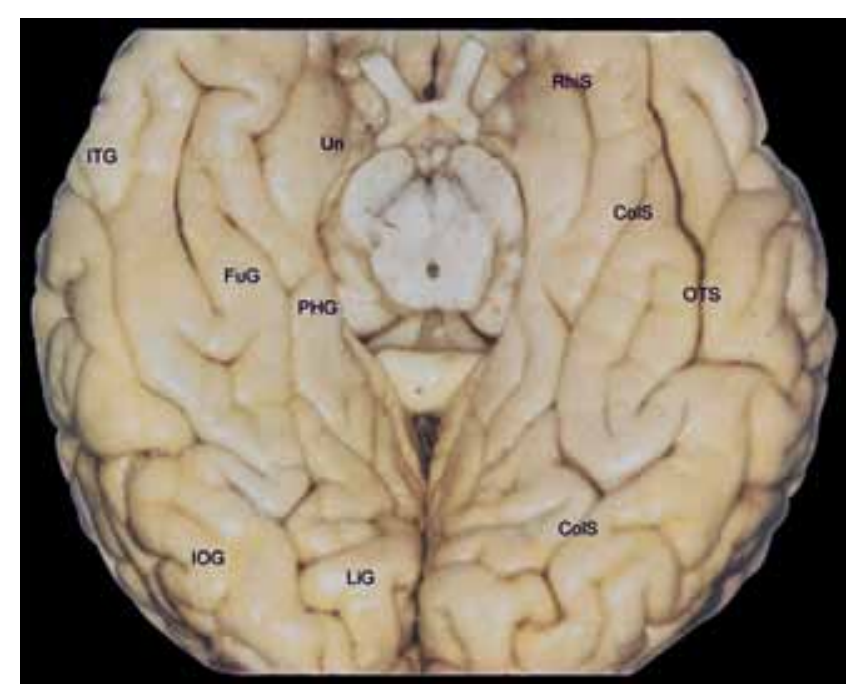

Fig. 10. The basal temporooccipital surface. ColS = collateral sulcus; FuG = fusiform gyrus; IOG = inferior occipital gyrus; ITG = inferior temporal gyrus; LiG = lingual gyrus; OTS = occipitotemporal sulcus; $\mathrm{PHG}=$ parahippocampal gyri; RhiS = rhinal sulcus; $U n=$ uncus.

Superiorly, the superior occipital gyrus extends along the superior border of the cerebral hemisphere, thus continuing along the medial surface to the cuneal gyrus. Inferiorly, the inferior occipital gyrus extends along the inferolateral margin, and its basal surface is lateral to the medial temporooccipital gyrus, also known as the lingual gyrus, as well as to the collateral sulcus that separates the two.

On the medial surface of the cerebral hemisphere, as previously mentioned, the occipital lobe is delineated and characterized by its well-defined and anatomically constant sulci and gyri. Its principal sulcus is the calcarine fissure, which is located just above the inferomedial margin of the cerebral hemisphere. The calcarine fissure starts over the splenium of the corpus callosum, delineating inferiorly the isthmus of the cingulate gyrus from the parahippocampal gyrus, and continues posteriorly as a gentle and superior convex curvature from whose apex emerges, superiorly, the parietooccipital sulcus, which in turn delineates anteriorly the occipital lobe on the medial surface of the cerebral hemisphere. Posteriorly, the calcarine fissure occasionally crosses the superomedial margin and extends along the occipital pole to the superolateral surface of the cerebral hemisphere.

The point of emergence of the parietooccipital sulcus divides the calcarine fissure into its proximal and distal parts. Superior to the proximal portion of the calcarine fissure and anterior to the parietooccipital sulcus is the precuneal gyrus, which is part of the parietal lobe. Superior to its distal part and posterior to the parietooccipital sulcus is the cuneus, or cuneal gyrus, so designated because of its wedge shape.

Inferiorly and along the entire length of the calcarine fissure is the medial temporooccipital gyrus, or lingual gyrus, which anteriorly continues to the parahippocampal gyrus and constitutes the mediobasal portion of the occipital lobe, which is supported on the cerebellar tentorium. 
The lingual gyrus is therefore delineated superiorly by the calcarine fissure and inferiorly by the collateral sulcus, which is a deep and generally continuous sulcus situated at the cerebral base, extending from the occipital pole to the temporal lobe and running parallel to the calcarine fissure.

The parietooccipital sulcus and calcarine fissure appear continuous on the surface. However, when their borders are retracted, it is obvious that they are separated by one or more small gyri. These gyri are composed of extensions of the cuneus and are known as the cuneolingual gyri.

The proximal part of the calcarine fissure is classified as a complete sulcus because its depth creates a rise in the medial wall of the occipital horn of the lateral ventricle, designated the calcar avis, and the distal part is considered an axial sulcus given that its axis runs along the visual cortex. Only the distal part includes the primary visual cortical areas, which are located on its superior (cuneal) and inferior (lingual) surfaces.

On the basal surface of the cerebral hemisphere, lateral to the lingual gyrus, is the medial temporooccipital gyrus or fusiform gyrus, situated between the collateral sulcus and the temporooccipital sulcus. The temporooccipital sulcus lies lateral and parallel to the collateral sulcus, rarely extends to the occipital pole, and in general is interrupted and divided into 2 or more parts. Anteriorly, the temporooccipital sulcus often bends medially and joins the collateral sulcus. The fusiform gyrus, in turn, extends to the basal surface of the temporal lobe, and lateral to its posterior portion lies the inferior occipital gyrus, whose lateral aspect constitutes the inferiormost portion of the lateral surface of the occipital lobe.

\section{The Temporal Lobe}

The temporal lobe is situated inferior to the sylvian fissure and delineated posteriorly by the imaginary line running from the superomedial portion of the parietooccipital sulcus to the preoccipital notch. Its lateral surface is composed of the superior, middle, and inferior temporal gyri-also respectively known as T1, T2, and T3which are separated by the superior and inferior temporal sulci, parallel to the lateral or sylvian fissure (Fig. 3). Anteriorly, the middle temporal gyrus is generally shorter, causing the superior and inferior temporal gyri to come together, and thereby forming the temporal pole.

The superior temporal sulcus is always a very well defined and deep sulcus and often presents as a continuous sulcus because of its parallelism with the sylvian fissure. In fact, in the past it was called the parallel sulcus. The inferior temporal sulcus is usually discontinuous and composed of various parts. Both of the temporal sulci start at the proximal portion of the temporal pole and end posterior to its borders. Whereas the posterior portion of the sylvian fissure typically terminates as an ascending curve that penetrates the supramarginal gyrus, the superior temporal sulcus always terminates at a point posterior to the end of the sylvian fissure (posterior sylvian point). In general, the superior temporal sulcus then bifurcates into an ascending sulcal segment, which separates the supramarginal gyrus from the angular gyrus,

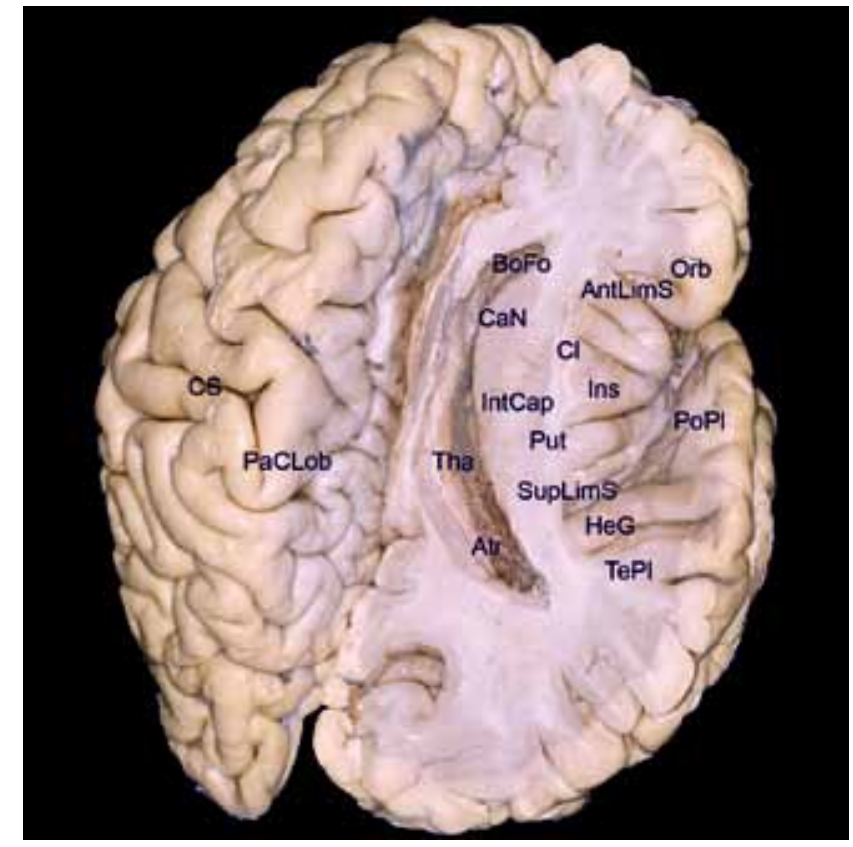

Fig. 11. The temporal opercular surface, the insula, and the central core of the brain. AntLimS = anterior limiting sulcus; Atr = atrium of lateral ventricle; $\mathrm{BoFo}=$ body of fornix; $\mathrm{CaN}=$ caudate nucleus; $\mathrm{Cl}$ = claustrum; $\mathrm{CS}$ = central sulcus; $\mathrm{HeG}$ = Heschl gyrus; Ins = insula; IntCap = internal capsule; Orb = orbital part of inferior frontal gyrus; PaCLob = paracentral lobule; PoPI = polar plane of the opercular temporal surface; Put = putamen; SupLimS = superior limiting sulcus of insula; $\mathrm{TePI}=$ temporal plane; $\mathrm{Tha}=$ thalamus.

and corresponds to the intermediate sulcus of Jensen, and a distal and horizontal segment that penetrates the angu-

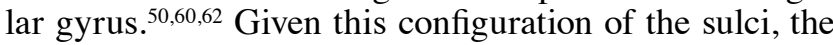
superior temporal gyrus always continues posteriorly to the supramarginal gyrus, encircling the terminal portion of the sylvian fissure. The middle temporal gyrus is often partially connected to the angular gyrus beneath the distal and horizontal portion of the superior temporal sulcus that penetrates the angular gyrus proper and inferiorly is often connected to the inferior occipital gyrus. In turn, the inferior temporal gyrus continues to the inferior occipital gyrus, over the preoccipital notch that posteriorly delineates the temporal lobe. Inferiorly, the inferior temporal gyrus extends along the inferolateral margin of the cerebral hemisphere. Medially, its basal surface lies along the lateral temporooccipital gyrus, or fusiform gyrus, and along the temporooccipital sulcus that separates the 2 gyri (Fig. 10).

The superior temporal gyrus constitutes the temporal operculum and covers the inferior aspect of the insular surface, and its superior, or opercular, surface (Fig. 11), which is within the sylvian fissure, is composed of various transverse gyri that emerge from the superior temporal gyrus and are directed obliquely toward the inferior part of the circular insular sulcus. ${ }^{73,78}$

Chief among these temporal gyri of the operculum is a much more voluminous transverse gyrus that originates in the posteriormost portions of the superior temporal gyrus and is oriented diagonally toward the posterior vertex of 

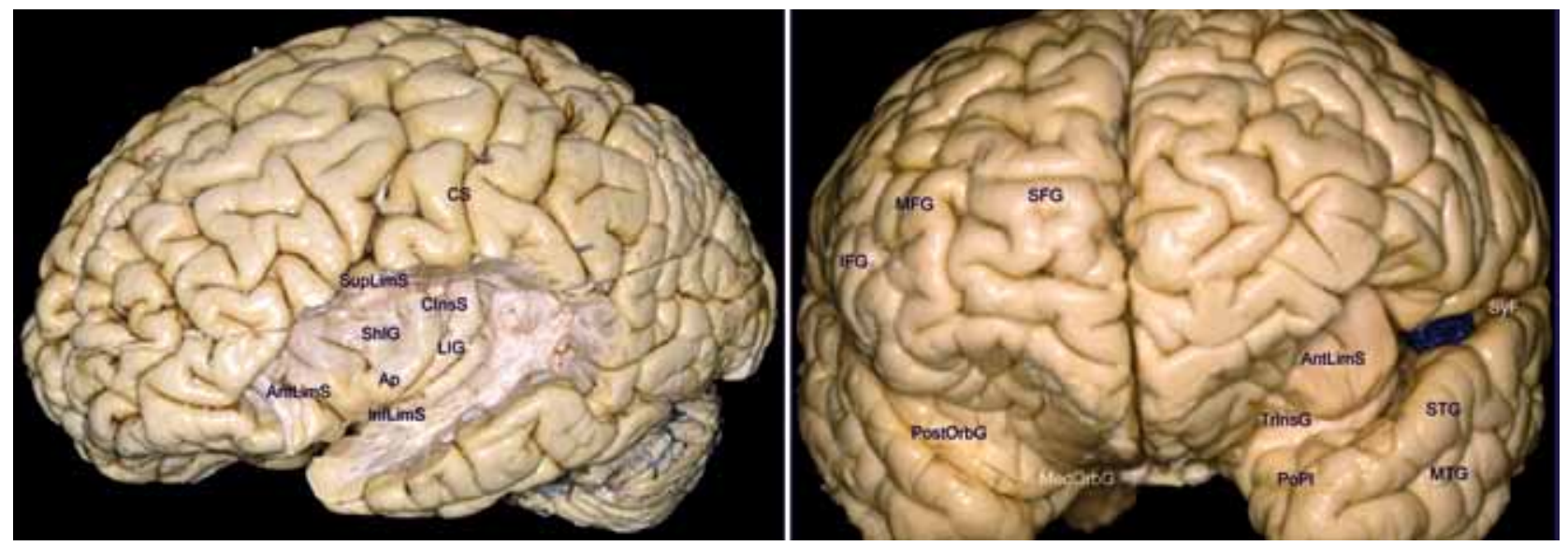

FIG. 12. The lateral (left) and anterior (right) surfaces of the insula. AntLimS = anterior limiting sulcus; Ap = insular apex; CInsS = central insular sulcus; CS = central sulcus; IFG = inferior frontal gyrus; InfLimS = inferior limiting sulcus; ITG = inferior temporal gyrus; LIG = long insular gyri; MedOrbG = medial orbital gyrus; MFG = middle frontal gyrus; MTG = middle temporal gyrus; $\mathrm{PoPI}=$ polar plane of the opercular temporal surface; PostOrbG = posterior orbital gyrus; SyF = sylvian fissure; SFG = superior frontal gyrus; ShIG = short insular gyri; STG = superior temporal gyrus; SupLimS = superior limiting sulcus of insula; TrlnsG = transverse insular gyrus.

the floor of the sylvian fissure and toward the ventricular atrium. This gyrus is designated the transverse gyrus of Heschl. In some brains, 1 or 2 sulci divide this gyrus; and in such cases, 2 or 3 gyri are also featured. Together with the posteriormost aspect of the superior temporal gyrus, the most anterior transverse gyrus of Heschl constitutes the primary auditory cortical area ${ }^{73,79}$ This gyrus has particular topographical importance because it underlies the opercular surface of the postcentral gyrus, its longest axis is oriented toward the ventricular atrium, and it divides the temporal opercular surface into 2 planes: an anterior plane called the polar plane and a posterior plane known as the temporal plane (Fig. 11). ${ }^{78}$

The floor of the polar plane is composed of short transverse gyri at oblique angles, and the lower border of the plane is defined by the inferior portion of the circular insular sulcus that runs along the bottom of the sylvian fissure. The temporal plane is triangular with an internal vertex that exactly corresponds to the posterior vertex at the bottom of the sylvian fissure, where the superior part of the circular insular sulcus comes into contact with its inferior part. The temporal plane is oriented horizontally and faces the inferior surface of the supramarginal gyrus as if supporting its anteriormost portion. Whereas the sylvian fissure appears oblique in coronal slices taken in the polar plane, it appears horizontal in those taken in the temporal plane.

The basal surface of the temporal lobe is continuous with the basal surface of the occipital lobe and is situated over the floor of the middle cranial fossa, anterior to the petrous portion of the temporal bone, whereas the basal occipital surface lies over the superior surface of the cerebellar tentorium.

The temporal lobe is composed laterally of the inferior surface of the inferior temporal gyrus and the anterior portion of the lateral temporooccipital gyrus, or fusiform gyrus, and medially of the inferior surface of the parahippocampal gyrus. The fusiform gyrus is situated lateral to the parahippocampal and lingual gyri, between the collat- eral and temporooccipital sulci. Its temporal portion presents a slight basal prominence due to its adaptation to the concavity of the middle cranial fossa. Its anterior aspect is typically curved or pointed, because the anteriormost portion of the temporooccipital sulcus frequently presents a medial curvature toward the collateral sulcus (Fig. 10). The anterior border of the fusiform gyrus, in general, corresponds medially to the level of the mesencephalic peduncle; as a whole, it constitutes the floor of both the atrium and the inferior horn of the lateral ventricle.

The parahippocampal gyrus and the anterior portion of the collateral sulcus have been described as limbic lobe structures.

\section{The Insular Lobe}

On the publication of the fourth edition of the Paris Nomina Anatomica in $1975,{ }^{18}$ the insula came to be considered a cerebral lobe. The insular surface is composed of the so-called mesocortex, which is anatomically situated between the allocortex, which is older and topographically more medial (comprising the amygdala and hippocampus), and the isocortex, which is phylogenetically younger and topographically more lateral (comprising the neocortex of the cerebral hemispheres).

Embedded between the frontal and temporal lobes of each cerebral hemisphere and constituting the base of each sylvian cistern, the insula has an anterior surface and a lateral surface (Fig. 12) that are encased in their respective opercular convolutions, ${ }^{73}$ which correspond to the regions of the cerebral hemispheres that have lately become more fully developed. The anterior surface of the insula is covered by the frontoorbital operculum (comprising the posterior portion of the posterior orbital gyrus and the orbital part of the inferior frontal gyrus), whereas its lateral surface is covered superiorly by the frontoparietal operculum (triangular and opercular parts of the inferior frontal gyrus, subcentral gyrus, and anterior and basal part of the supramarginal gyrus) and inferiorly by 


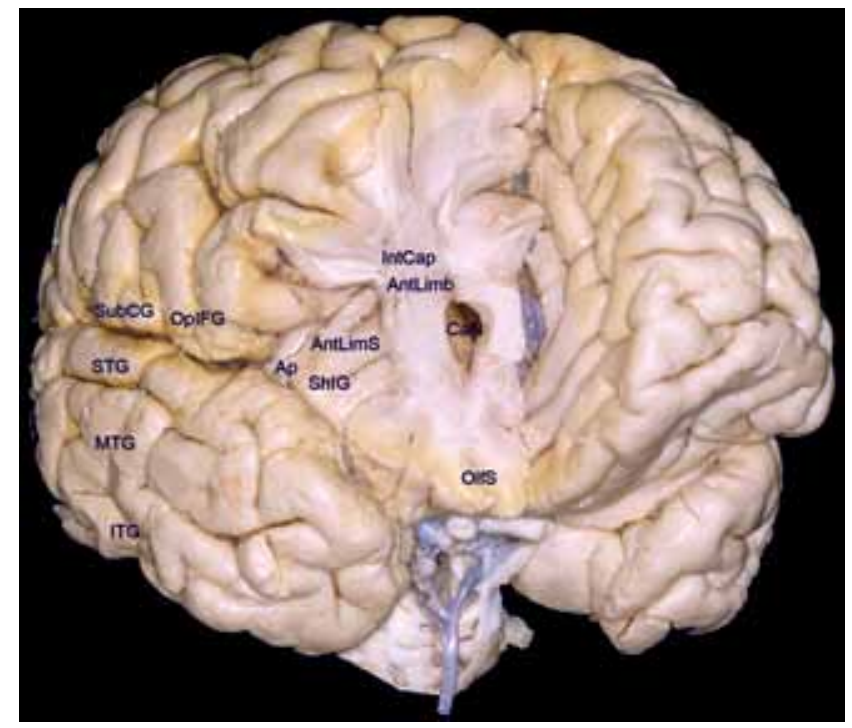

FIG. 13. The anterior surface of the insula and the anterior horn of the lateral ventricle. AntLimb = anterior limb of the internal capsule; AntLimS = anterior limiting sulcus; $\mathrm{Ap}=$ insular apex; $\mathrm{CaN}=$ caudate nucleus; IntCap = internal capsule; ITG = inferior temporal gyrus; MTG = middle temporal gyrus; OlfS = olfactory sulcus; OpIFG = opercular part of the inferior frontal gyrus; ShIG = short insular gyri; STG = superior temporal gyrus; SubCG = subcentral gyrus.

the temporal operculum (superior temporal gyrus; Figs. 5 and 6$)^{73,79,87}$

The lateral surface of the insula is characterized as a pyramid with a triangular base, whose anteroinferior vertex constitutes the limen insulae, and is divided by the oblique central sulcus of the insula into an anterior portion and a posterior portion, with the former having a larger area. The anterior portion is typically composed of 3 short gyri that originate at the apex of the insula, which corresponds to the most prominent aspect of the insular pyramid, with the anteriormost aspect extending over the anterior surface of the insula; and the posterior portion, in general, is composed of 2 long gyri not originating at the apex but oriented obliquely and in parallel. The transverse and accessory insular gyri, which together constitute the insular pole, also originate from the apex of the insula. ${ }^{73,74}$ The transverse insular gyrus, which is situated more inferiorly, runs along the limen insulae and is connected to the posteromedial orbital lobule, which is composed of the posterior portion of the medial orbital gyrus and the medial portion of the posterior orbital gyrus ${ }^{73,81}$ and is located anterior and along the lateral olfactory stria.

The insular surface is delineated peripherally by the circular sulcus of Reil, ${ }^{14,70}$ or periinsular sulcus, ${ }^{72,73}$ which is interrupted by the previously mentioned transverse insular gyrus. Given the triangular shape of the insula, its circular, or periinsular, sulcus is usually divided into 3 parts, that is, the anterior, superior, and inferior periinsular sulci, ${ }^{73}$ also designated the anterior, superior, and inferior limiting sulci of the insula.$^{57}$

To understand the periinsular spaces more fully, one should remember that the insula has a lateral surface and an anterior surface. The superior and inferior limiting sulci are morphologically categorized as true sulci that

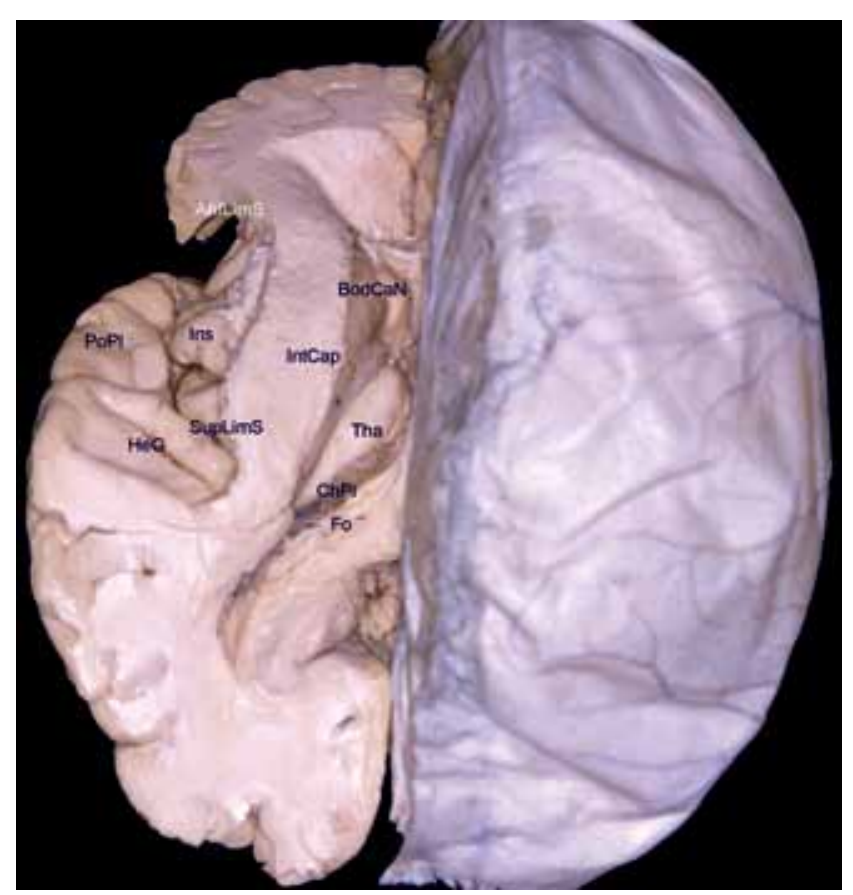

FIG. 14. The insular lateral surface corresponds to an external shield of the central core of the brain. AntLimS = anterior limiting sulcus of insula; BodCaN = body of the caudate nucleus; $\mathrm{ChPI}=$ choriodal plexus; Fo = fornix; HeG = Heschl gyrus; Ins = insula; IntCap = internal capsule; PoPI = polar plane of the opercular temporal surface; SupLimS = superior limiting sulcus of insula; Tha $=$ thalamus.

delineate the respective transitions and deflections occurring among the lateral insular surface and the frontoparietal operculum, and the lateral insular surface and the temporal operculum. The so-called anterior limiting sulcus of the insula, on the other hand, is considerably deeper and morphologically characteristic of a true fissure or space that separates the anterior surface of the insula from the posterior surface of the posterior orbital gyrus.

The upper half of the fundus of the anterior limiting sulcus is separated from a true anterior ventricular recess at the head of the caudate nucleus only by the fibers of the thin anterior limb of the internal capsule, whereas the fundus of the lower half continues to the ventral striatopallidal region (Fig. 13).

From a morphological and topographical perspective, the surface of the insula clearly represents the external shield of a true central core of the brain, ${ }^{57}$ quite well defined anatomically. This central core of the brain comprises, in each cerebral hemisphere, the insula proper, the basal nuclei, the thalamus, and the internal capsule (Fig. 14). The anterior half of the lateral surface of the insula corresponds internally to the head of the caudate nucleus, whereas the posterior half corresponds to the thalamus and the body of the caudate nucleus. Each central core of each cerebral hemisphere, composed of all the structures mentioned above, is incorporated into the corresponding half of the mesencephalon, morphologically characterizing a brainstem with 2 heads that correspond to the 2 central cores (Fig. 15).

Under the insular cortex and its respective subcorti- 


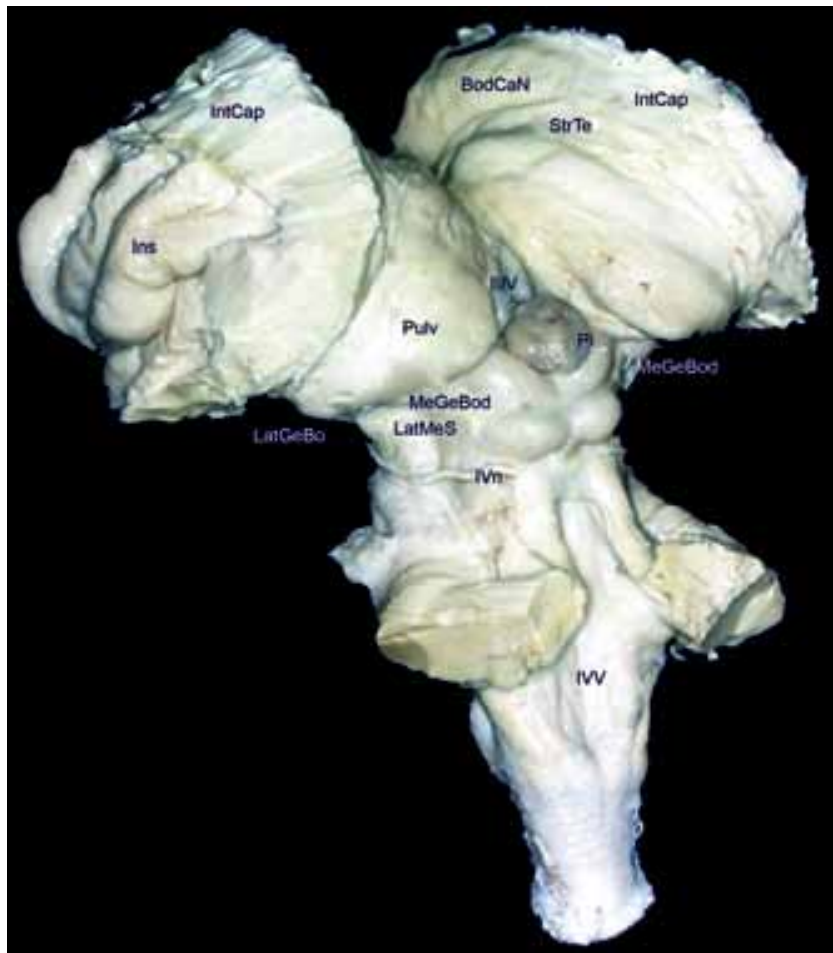

FIG. 15. Each central core of the brain corresponds to a head of each half of the brainstem. BodCaN = body of the caudate nucleus; Ins = insula; IntCap = internal capsule; LatGeBo = lateral geniculate body; LatMeS = lateral mesencephalic sulcus; $\mathrm{MeGeBod} \mathrm{=} \mathrm{medial} \mathrm{geniculate}$ body; $\mathrm{Pi}=$ pineal gland; $P$ Pulv = pulvinar of thalamus; $\mathrm{StrTe}=$ stria terminalis; IIIV = third ventricle; IVn = trochlear nerve; IVV = fourth ventricle.

cal white matter, also known as the extreme capsule, is the fine lamina of gray matter that constitutes the claustrum. Underneath that is the putamen, enclosed within its external and internal capsules. The thin external capsule is composed of fibers that cover only the lateral portion of the putamen and are bereft of major functional importance, whereas the internal capsule consists of important projection fibers that originate from, and are directed toward, the cerebral cortex as a whole. Since the area of the putamen is slightly smaller than that of the lateral surface of the insula, ${ }^{73}$ and given the shape of the internal capsule, the recesses of the anterior, superior, and inferior limiting sulci are practically adjacent to the fibers of the internal capsule.

\section{The Limbic Lobe and Correlated Areas}

The Limbic Lobe. The publication entitled International Anatomical Terminology, published in $1998^{21,66}$ and replacing the previous Nomina Anatomica, introduced the limbic lobe as another of the cerebral lobes and described it as comprising the cingulate and parahippocampal gyri.

The term limbic was first used in the 19th century by Broca, ${ }^{5}$ who observed that certain cerebral structures constituted a continuum arranged in the shape of a $\mathrm{C}$ surrounding the diencephalic region. Since then the term limbic - meaning border, ring, or surround ${ }^{22}$ - came to be definitively established in the neuroanatomical literature.
TABLE 3: Principal limbic cortical structures

\author{
cingulate gyrus \\ parahippocampal gyrus \\ hippocampal formation \\ hippocampus (Ammon's horn) \\ subiculum \\ dentate gyrus \\ prehippocampal rudiment/indusium griseum \\ frontal mediobasal cortical area \\ paraterminal gyrus \\ paraolfactory gyri or subcallosal area \\ olfactory cortical areas
}

Subsequent studies introduced the notion that the limbic system is composed of telencephalic and diencephalic structures that, despite their anatomical and functional diversity, are particularly responsible for the physiology of emotions, memory, and learning. . $9,33,58,59,79^{2}$

The limbic system, in its entirety, is composed of cortical, subcortical, and nuclear structures that are interconnected and have connections with other areas of the CNS via a complex network of tracts (Table 3 ). However, the principal elements of the limbic system are the hippocampal formation and the amygdala, which participate in distinct circuits with the rest of the brain. The hippocampal formation is principally related to telencephalic and diencephalic structures via circuits whose basic purpose is to convert short-term memory into long-term memory, whereas the circuits that include the amygdala are more strictly related to the emotions and ultimately influence the effector systems (autonomic, neuroendocrine, and motor), primarily via the hypothalamus.

Within the medial surface of each cerebral hemisphere, one prominent feature is the cingulate gyrus, which wraps around the corpus callosum and continues posteriorly and inferiorly to the parahippocampal gyrus, forming the shape of a $\mathrm{C}$ around the diencephalon (Fig. 4). Broca originally described the cingulate and parahippocampal gyri as contiguous, jointly dubbing them the greater limbic lobe. He also considered the cingulate, subparietal, and collateral sulci to be segments of the sulcus he referred to as the limbic sulcus. ${ }^{5,29,30}$

The cingulate gyrus is situated above the callosal sulcus and below the cingulate sulcus. It starts below the rostrum of the corpus callosum, and as it ascends around the knee of the corpus callosum, it typically presents a connection with the medial (or medial aspect of the superior) frontal gyrus. Under the trunk of the corpus callosum it is connected to the paracentral lobule, and more posteriorly it is connected to the precuneus. These connections, which vary in number, are arrayed from front to back and from bottom to top and are particularly visible after removing the cingulate gyrus's most cortical aspect.

Posterior to the splenium of the corpus callosum 


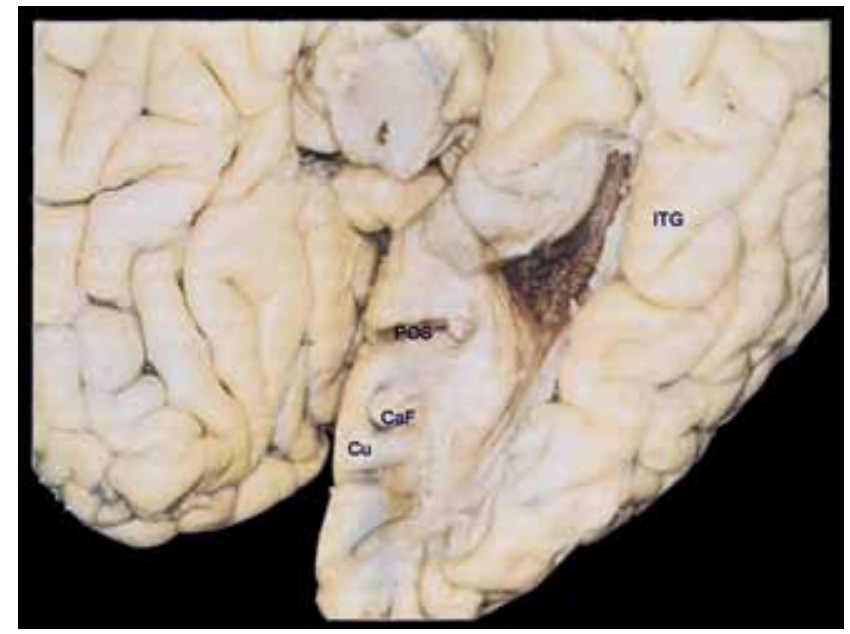

Fig. 16. Basal view of the parahippocampal gyrus and the atrium after removing the fusiform and lingual gyri. $\mathrm{CaF}=$ calcarine fissure; $\mathrm{Cu}$ = cuneus; ITG = inferior temporal gyrus; POS = parietooccipital sulcus.

the cingulate gyrus consistently becomes narrower, at which point it is referred to as the isthmus of the cingulate gyrus, and continues to the parahippocampal gyrus. The site of transition between these 2 gyri is identified by the emergence of the anterior branch of the calcarine fissure, which originates beneath the isthmus of the cingulate gyrus.

As already mentioned, the terminal ascending branch of the cingulate sulcus delineates posteriorly the paracentral lobule and anteriorly the precuneus, whereas the subparietal sulcus is located inferior to the precuneus, separating it from the cingulate gyrus and appearing to be a posterior continuation of the cingulate sulcus after a short interruption of the latter. The connections between the cingulate and the precuneus gyri are anterior and posterior to the subparietal sulcus.

The parahippocampal gyrus forms the lower half of the $C$ that wraps around the diencephalic region. Posteriorly, it comprises the isthmus of the cingulate gyrus and is also the anterior continuation of the lingual gyrus, which lies under the calcarine fissure. The parahippocampal gyrus is situated lateral to the cerebral peduncle. Anteriorly, it folds back on itself medially, assuming the shape of a hook and constituting the uncus of the parahippocampal gyrus situated anterolateral to the cerebral peduncle, and harbors the uncal sulcus.

Medially, the parahippocampal surface curves superiorly and laterally, constituting the subiculum, characterized as a flat, superior surface running along the anteroposterior axis of the parahippocampal gyrus and under the pulvinar of the thalamus, so that these 2 surfaces constitute the portion of the transverse fissure of the brain that harbors the so-called wing of the ambient cistern. The hippocampus is situated lateral to the subiculum.

The hippocampus consists of Ammon's horn, which is characterized as an intraventricular prominence (Fig. 16), and the small dentate gyri, which lie laterally along Ammon's horn. The small dentate gyri are separated from the subiculum by the hippocampal sulcus, which anteriorly terminates within the uncus. Given the greater

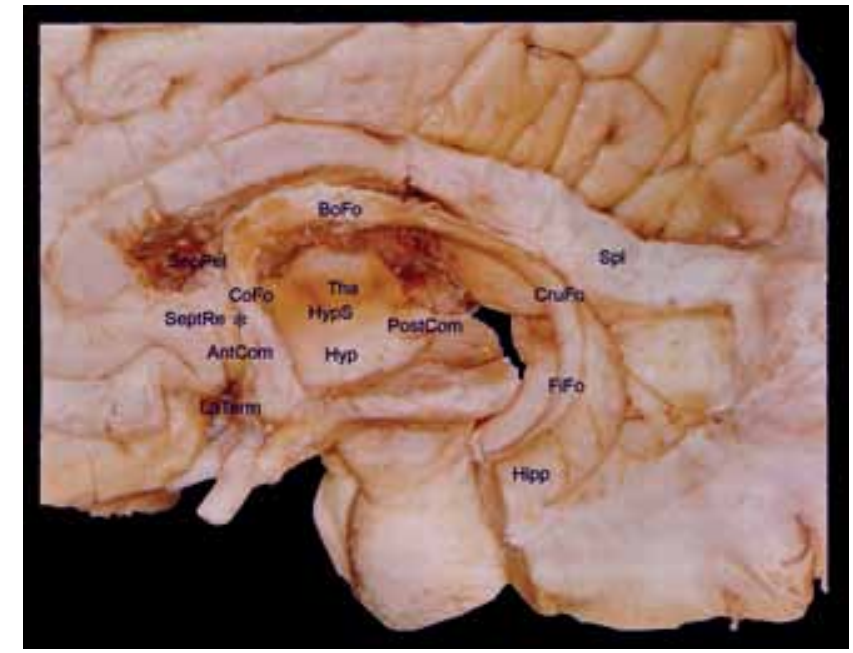

Fig. 17. The corpus callosum, fornix, septal area, and septum pellucidum. AntCom = anterior commissure; BoFo = body of fornix; CoFo $=$ column or pillar of the fornix; CruFo = crura of fornix; FiFo = fimbria of the fornix; Hipp = hippocampus; Hyp = hypothalamus; HypS = hypothalamic sulcus; LaTerm = lamina terminalis; PostCom = posterior commissure; SepPel = septum pellucidum; SeptRe = paraterminal gyrus/septal region; $\mathrm{Spl}=$ splenium of corpus callosum; Tha $=$ thalamus .

magnitude of Ammon's horn, the term hippocampus is commonly used in reference to this structure. Within the ventricular cavity, Ammon's horn, or the hippocampus, is covered by the alveus, a thin layer of fibers that gives rise to the fimbria of fornix, the principal bundle of efferent fibers of the hippocampus (Fig. 17). These structures are collectively known as the hippocampal formation. Between the dentate gyri and the fimbria of fornix is the fimbriodentate sulcus, which lies lateral and parallel to the hippocampal sulcus. ${ }^{15,78}$

The uncus of the parahippocampal gyrus is triangular with a medial vertex, such that its anteromedial surface faces the carotid cistern and its posteromedial surface faces and encircles the mesencephalic peduncle (Fig. 7). Two small prominences known as the semilunar gyrus and ambient gyrus, which are separated by the semiannular sulcus, are evident in the anterior portion of the surface of the uncus. The ambient gyrus, which is more inferior, often presents with a depression caused by the pressure of the free edge of the tentorium cerebelli. The anterior half of the uncus includes the amygdala, whereas its posterior half includes the head of the hippocampus. ${ }^{78}$ Superiorly, the amygdala runs toward the base of the globus pallidus so that, in a coronal slice, the base of the lentiform nucleus and the amygdala form a figure-eight or an hourglass shape. ${ }^{74,78,79}$

Along the cerebral base, the parahippocampal gyrus is laterally delineated by the collateral sulcus, which separates it from the fusiform gyrus, and by the rhinal sulcus, which is occasionally continuous with the collateral sulcus. The rhinal sulcus, which is not always readily identifiable, is consistently the sulcus that separates the uncus from the rest of the temporal pole (Figs. 7 and 10).

Some authors consider the indusium griseum and its connections as also belonging to the hippocampal formation. ${ }^{15,79}$ The indusium griseum is composed of a thin 
layer of gray matter situated over the corpus callosum. On each side, the indusium griseum is characterized by medial and lateral striae that run within the callosal sulcus and constitute the remains of the vestigial indusium. Because it lies laterally within the callosal sulcus, it can be confused with the cortex of the cingulate gyrus of each side. Anteriorly, the indusium griseum is connected to the paraterminal gyrus via the prehippocampal rudiment. Posteriorly, it circles the splenium of the corpus callosum and on each side runs along the fasciolar gyri (fasciola cinerea, gyrus of Andreas Retzius, and retrosplenial gyri), a thin layer of gray matter that reaches to the posterior portion of the dentate gyrus.

To ease understanding of the anatomical features, it is interesting to remember that, phylogenetically, the hippocampus is supracallosal in origin, subsequently migrating posteriorly and inferiorly and finally presenting as a structure that runs along the floor of the inferior horn of the lateral ventricle. ${ }^{64}$ Considering these observations, the indusium griseum can be understood as a remnant of the supracallosal hippocampus, and the fornix as its tail that was left behind during its inferior migration.

The Temporal Stem. Laterally, the parahippocampal gyrus is contiguous with the fusiform gyrus and the remainder of the basal surface of the brain. Posteriorly, it continues along the cingulate gyrus. Medially, it runs under the thalamus along the natural space comprising the choroidal fissure. Anteriorly, its uncal portion is superiorly incorporated into the lateralmost aspect of the frontobasal region via a well-defined neural peduncle anterior to the inferior horn of the lateral ventricle.

Anteriorly and externally, this true temporal peduncle is composed of the cortex of the transverse insular gyrus, which crosses the limen insulae, connecting to the posteromedial orbital lobule. ${ }^{82}$ Internally, it consists of the uncinate fascicle, which joins the frontal and temporal lobes: ${ }^{16}$ fibers of the frontooccipital fascicle, which are arrayed immediately posterior to the uncinate fascicle; amygdalofugal fibers, which are composed of the ventral extensions of the amygdala-that project to the septothalamohypothalamic region ${ }^{79}$ and the nucleus of the stria terminalis, situated under the head of the caudate nucleus ${ }^{29,30,40}$ - and fibers of the anterior commissure. Medially, it includes the superior extension of the amygdala, which superiorly extends medially to the putamen and toward the globus pallidus. ${ }^{78}$

In the literature, this set of structures has been given the generic and controversial name of temporal stem, equivalent to the temporal axis. According to Duvernoy, ${ }_{1}^{14}$ "the temporal stem consists of only a thin layer of white matter situated between the ventricular cavity and the fundus of the superior temporal sulcus." In contrast, Wen et al ${ }^{78}$ stated that the term refers "only to the connections between the temporal lobe and the insula, excluding the superior extension of the amygdala in the direction of the globus pallidus and the limen insulae." Türe et al. ${ }^{73}$ defined the temporal stem as "the portion of white matter that penetrates the temporal lobe between the anterior border of the insula and the inferior horn, . . . composed of the fronto-occipital fascicle and the anterior thalamic peduncle," and therefore being the portion anterior to the sagittal stratum, which in the present text is designated the temporal stem and can be understood as the peduncular portion of the temporal stem.

Posterolaterally, the temporal stem is contiguous with the layers of fibers called the sagittal strata, 37,74 which cover the inferior horn and ventricular atrium. All of these structures collectively join the temporal lobe to the remainder of the cerebral hemisphere beneath the insula. The so-called sagittal stratum consists of the frontooccipital fascicle, the inferior thalamic peduncle, or radiation-which encompasses the auditory and optic radiations-and the fibers that compose the anterior commissure and tapetum. The layer of callosal fibers known as the tapetum lies under the optic radiation and then constitutes the most inferior layer of the sagittal stratum. The sagittal stratum is situated inferiorly to the inferior limiting sulcus of the insula, forming the roof and lateral wall of the inferior horn and consituting the lateral wall of the ventricular atrium. Superficial to the sagittal stratum is the subcortical white matter of the entire neocortical portion of the temporal lobe.

From a topographical perspective, it is notable that the sagittal stratum corresponds to the set of fibers that cover the inferior horn and atrium of the ventricle, inferiorly and posteriorly to the insula, whereas the temporal stem is situated anterior to the inferior horn, connecting the anteromedial temporal portion to the basolateral frontal portion of the brain.

The Basal Forebrain and Ventral Striatopallidal Region. The mediobasal frontal cortical area of each cerebral hemisphere, composed of the paraterminal gyrus and the paraolfactory gyri, is also considered a limbic cortical area. The paraterminal gyrus is situated on the medial wall of each cerebral hemisphere, immediately facing and quasi-continuous with the lamina terminalis, and is delineated anteriorly by a short, vertical sulcus known as the posterior olfactory sulcus. The small anterior curvature of the paraterminal gyrus is called the prehippocampal rudiment and extends superiorly, along the previously described indusium griseum. Inferiorly, the paraterminal gyrus extends along the diagonal band of Broca and the lateral olfactory stria.

The posterior and anterior paraolfactory gyri, which are also vertical and separated by the anterior paraolfactory sulcus (the latter not always identifiable), are located anterior to the paraterminal gyrus. This area of the paraolfactory gyri is also known as the subcallosal area. Anterior to the subcallosal area is a fold that connects the basalmost portion of the cingulate gyrus with the gyrus rectus, encircles the posteriormost part of the superior rostral sulcus, and is called the cingulate pole..$^{82}$

The paraterminal gyri harbor the septal nuclei ${ }^{79}$ and constitute the septal area, which corresponds to the socalled paraolfactory area of Broca. ${ }^{36}$ Therefore, the septal region is situated on the medial surface of the cerebral hemisphere, immediately facing the anterior commissure. This region is also known as the septum verum ("true septum"), or precommissural septum, ${ }^{8,29,79}$ in counterpoint to the septum pellucidum, which does not contain neuronal 
cells and is situated posterior and superior to the anterior commissure, morphologically constituting the medial walls of the anterior horns and the ventricular bodies. (The septal nuclei receive afferents principally from the hippocampus and subiculum of the parahippocampal gyrus via the indusium griseum as well as from the precommissural fornix. The efferents of the septal nuclei project to the area surrounding the hippocampus via the fornix itself and to the hypothalamus, the mesencephalon [via the medial prosencephalic fasciculus], and the habenula [via the stria medullaris thalami], which in turn projects to the mesencephalon..$^{8,29,39,79}$ Functionally, the septal nuclei are responsible for connecting limbic structures with the hypothalamus and the brainstem, principally via the hippocampal formation. The so-called septal syndrome is clinically characterized by exaggerated reactions to environmental stimuli and the consequent behavioral alterations, principally related to eating and drinking habits, as well as by episodes of rage and disorders in the sexual sphere.)

The generic term olfactory cortical areas refers to the olfactory nerves, bulb, tract, trigone, and striae as well as the anterior perforated substance, the diagonal band of Broca, and the piriform lobe, in each cerebral hemisphere. The piriform lobe comprises the following: 1) the prepiriform cortical area; 2) the lateral olfactory stria, which extends to the gyrus semilunaris; 3 ) the uncus of the parahippocampal gyrus and the small gyri of which it consists (uncinate gyrus, caudal portion of the dentate gyrus or band of Giacomini, and intralimbic gyrus); and 4) the entorhinal area (rostralmost area of the parahippocampal gyrus, easily recognized by its speckled, superficial aspect, attributed to the discontinuity of its most superficial cell layer, characterized by islands of large, multipolar neurons).

The area known as the anterior perforated substance constitutes a particularly important topographical region of the basal forebrain (Fig. 7 right). Macroscopically, this area is delineated anteriorly by the olfactory trigone and the lateral and medial olfactory striae, whereas it is delineated posteriorly by the edges of the optic tracts, medially by the interhemispheric fissure, and laterally by the uncus of the parahippocampal gyrus and the limen insulae. Topographically, the anterior perforated substance is situated just above the bifurcation of the internal carotid artery, and thus forming the roof of the space that harbors the distal portion of the artery and the proximal segments of the anterior and middle cerebral arteries. The perforating branches that emerge from those arterial segments constitute the lenticulostriate arteries, and it is from the surface of the anterior perforated substance that they penetrate the frontobasal parenchyma. In fixed anatomical specimens from which the arachnoid and blood vessels have been removed, its surface is easily identified by its multiple orifices. Laterally, the anterior perforated substance continues to the limen insulae, where it extends along the prepiriforme cortex (the cortical area that lies lateral to the lateral olfactory stria and is also occasionally referred to as the lateral olfactory gyrus). More posteriorly, it extends to the periamygdaloid area (the semilunar gyrus, where the lateral olfactory stria terminates

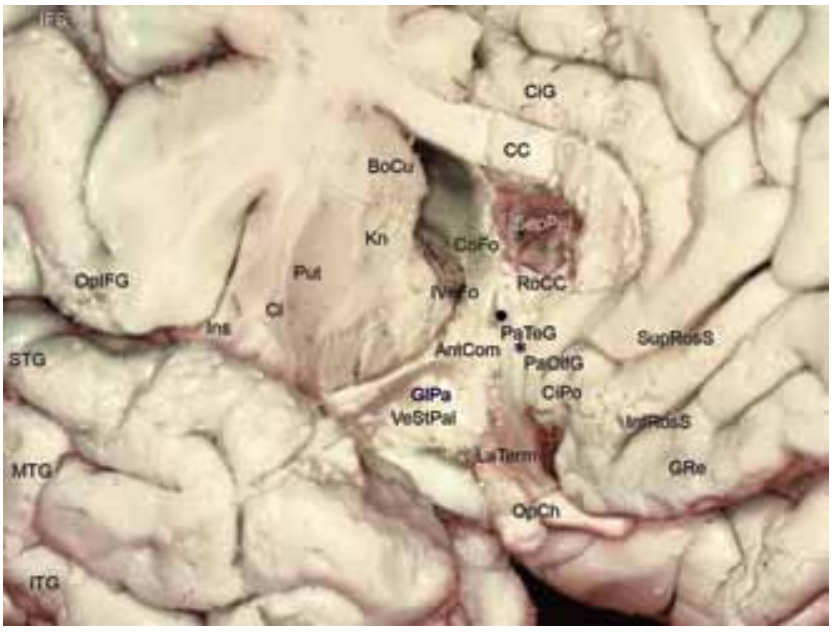

Fig. 18. The subinsular central core structures and the ventral striatopallidal and septal region. AntCom = anterior commissure; $\mathrm{BoCu}=$ body of caudate nucleus; $\mathrm{CC}=$ corpus callosum; $\mathrm{CiG}=$ cingulate gyrus; $\mathrm{CiPo}=$ cingulate pole; $\mathrm{Cl}=$ claustrum; $\mathrm{CoFo}=$ column or pillar of the fornix; $\mathrm{GIPa}=$ globus pallidus; $\mathrm{GRe}=$ gyrus rectus; IFS = inferior frontal sulcus; InfRosS = inferior rostral sulcus; Ins = insula; ITG = inferior temporal gyrus; IVeFo = interventricular foramen of Monro; $\mathrm{Kn}=$ knee of corpus callosum; LaTerm = lamina terminalis; MTG = middle temporal gyrus; OpCh = optic chiasm; OpIFG = opercular part of the inferior frontal gyrus; $\mathrm{PaOlfG}=$ paraolfactory gyri; $\mathrm{PaTeG}=$ paraterminal gyrus; $\mathrm{Put}$ = putamen; RoCC = rostrum of the corpus callosum; SepPel = septum pellucidum; STG = superior temporal gyrus; SupRosS = superior rostral sulcus; VeStPal = ventral striatopallidal region.

and which harbors the cortical amygdaloid nuclei of the amygdaloid complex). The posteriormost portion of the anterior perforated substance is traversed by the diagonal band of Broca, a particularly smooth bundle of fibers immediately facing the optic tract.

Posteriorly, the anterior perforated substance extends along the cell aggregates and nerve fibers that compose the so-called ventral striatum region, which harbors the ventral striatopallidal system structures and is currently considered an important site in relation to the limbic system. From a functional point of view, one of the basic principles of the concept of the limbic system is that its structures project to the hypothalamus-not to the basal ganglia, as occurs in the remainder of the cerebral cortex..$^{29,30,33}$ However, it has been shown that the portion most anterior and basal to the striatum (nucleus accumbens and striatal areas of the olfactory tubercle) receives projections not only from the olfactory cortex, hippocampus, entorhinal cortices, cingulate gyrus, and temporal pole, but also from the orbitomedial and insular cortices. In addition, the nucleus accumbens projects to the anteriormost portions of the globus pallidus, which in turn gives rise to anterior thalamocortical projections. These findings prompted Heimer and colleagues ${ }^{31,32}$ to include within the concept of the "greater limbic lobe" the posterior regions of the orbital cortex and the insula. Various authors came to view the ventral striatum and ventral pallidum as regions of the basal ganglia that include the ventral corticostriatopallidal system, which is distinct from the classical dorsal corticostriatopallidal system related to motor activities, and with particular 
neuropsychiatric implications. ${ }^{30,31,33,42}$ Functionally, the caudal, or dorsal, system is composed of certain cortical surface connections (neocortex-dorsal striatum-dorsal pallidum-ventrolateral thalamic nuclei-motor cortex), whereas the ventral system is composed of the allocortex and mesocortex connections (greater limbic lobe-ventral striatum-ventral pallidum-dorsomedial thalamic nuclei-prefrontal cortex/cortex of the anterior part of the cingulate gyrus).$^{29}$

The brain region currently known as the ventral striatopallidal system,,$^{29,32}$ or more simply the ventral striatum (Fig. 18), corresponds in part to the substantia innominata, a name taken from the historical literature in German, and can be confused with the concept of the basal forebrain in current English literature. (Note that the substantia innominata of Reichert corresponds most closely to the ventropallidal region. ${ }^{30,36}$ ) The ventral striatum refers to the basal forebrain region situated between the anterior perforated substance and the anterior commissure of each cerebral hemisphere. Superiorly, it is closely related to the most anterobasal portion of the anterior limb of the internal capsule, whereas laterally it is contiguous with the peduncle of the temporal stem, and medially it is particularly related to the septal region and the hypothalamus. The ventral striatopallidal region includes the nucleus accumbens, which corresponds to a basal connection of the head of the caudate nucleus with the most anterior and inferior portion of the putamen (hence the name ventral striatum), the globus pallidus, the magnocellular nucleus of the basal forebrain (nucleus basalis of Meynert), and the fibers that constitute the ventral extension of the amygdala and are directed toward the septal region, the hypothalamus, the thalamus, and the bed nucleus of the stria terminalis, located under the head of the caudate nucleus. Because of its topography, the ventral striatum is crisscrossed by the perforating lenticulostriate arteries. Functionally, the ventral striatum is closely correlated with neuropsychiatric functions. ${ }^{30,31,33,59}$

Superiorly, the ventral striatum and pallidum are covered by the anterior limb of the internal capsule, which harbors the fibers that carry their projections to the prefrontal and anterior cingulate cortices. ${ }^{32,40}$ Medial to the ventral striatum and ventral pallidum is the portion of the hypothalamus that forms the inferior part of the ipsilateral wall of the third ventricle and, more anteriorly, the septal region.

Topographical Aspects of the Limbic Structures. It is interesting to note that the mediobasal frontal cortical areas (paraterminal gyrus and subcallosal area), the olfactory cortical areas (anterior perforated substance and components of the piriform lobe), and the ventral pallidal-striatum region (with its subjacent nuclei) constitute a corticosubcortical continuum running along the ventral surface of the brain from the medial portion of the temporal pole to the posterior mediobasal portion of the frontal lobe, with its posterior border being delineated by the anterior commissure.

These structures extend along the anterior and medial surface of the cerebral hemisphere via the cingulate gyrus and along the basal surface via the parahippocam-

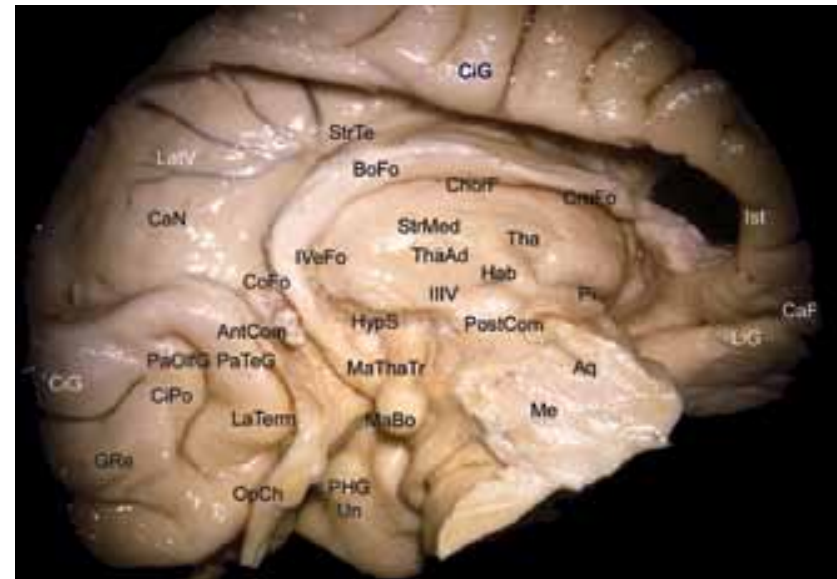

Fıg. 19. The medial deep cerebral structures after removal of the corpus callosum. AntCom = anterior commissure; $\mathrm{Aq}=$ cerebral aqueduct; $\mathrm{BoFo}=$ body of fornix; $\mathrm{CaF}=$ calcarine fissure; $\mathrm{CaN}=$ caudate nucleus; $\mathrm{ChorF}=$ choroidal fissure; $\mathrm{CiG}=$ cingulate gyrus; $\mathrm{CiPo}=$ cingulate pole; $\mathrm{CoFo}=$ column or pillar of the fornix; CruFo = crura of fornix; $\mathrm{GRe}=$ gyrus rectus; $\mathrm{Hab}=$ habenula; HypS = hypothalamic sulcus; Ist = isthmus of cingulate gyrus; IVeFo = interventricular foramen of Monro; LaTerm = lamina terminalis; LatV = lateral ventricle; LiG = lingual gyrus; $\mathrm{MaBo}=$ mamillary body; MaThaTr $=$ mamillothalamic tract; $\mathrm{Me}=$ mesencephalon; OpCh = optic chiasm; PaOlfG = paraolfactory gyri; PaTeG = paraterminal gyrus; $P H G$ = parahippocampal gyri; $\mathrm{Pi}=$ pineal gland; PostCom = posterior commissure; StrMe = stria medullaris of thalamus; StrTe = stria terminalis; Tha = thalamus; ThaAd = interthalamic adhesion; Un = uncus; IIIV = third ventricle.

pal gyrus; these 2 gyri are posteriorly continuous (Fig. 19). Therefore, the limbic lobe looks like a slightly tilted circle. In relation to the midsagittal plane, in which the diencephalic structures are located, the superior portion of the limbic lobe is more medial, whereas its inferior portion is more lateral. The ventral striatopallidal region, which encompasses the substantia innominata, is then situated under the anterior base of the limbic lobe. Topographically, it is posterior to the anterior perforated substance, anterior to the anterior commissure, and inferior to the fibers of the anterior limb of the internal capsule. Laterally, the ventral striatum is contiguous with the temporal stem and the amygdaloid complex, whereas medially it is contiguous with the septal region.

In parallel with these observations, Mesulam ${ }^{43}$ proposed that because of their particularly superficial presentation, the most medial portions of the amygdaloid complex, the substantia innominata (within the ventral striatopallidal region), and the septal nuclei (within in the paraterminal gyri), which together constitute the basal forebrain, should be considered constituents of the cerebral mantle.

In conclusion, it should be emphasized that although the cortical areas mentioned compose the cortical portion of the limbic lobe, the concept of the limbic system as a functional unit also involves the participation of deep structures and is controversial in terms of its conception and composition. ${ }^{29-31,59}$ From a morphological perspective, however, the structures that make up the limbic system present as a series of C-shaped curves centered around the thalamus and hypothalamus in each cerebral hemisphere. Nieuwenhuys et al. ${ }^{45}$ characterized the orga- 
nization (medial to lateral) of 5 of these curves: 1) stria medullaris thalami, habenular nuclei, and habenulointerpeduncular tract; 2) amygdala, posterior extension of the amygdala (stria terminalis), and anterior extension of the amygdala, which project to the nucleus of the stria terminalis, located below the head of the caudate nucleus, and together constitute the so-called extended amygdala; $;{ }^{48,49} 3$ ) fimbria, crura, body, and column of fornix, which connect the hippocampus to the mammillary body; 4) hippocampus and longitudinal striae (indusium griseum), which connect the hippocampus with the paraterminal gyrus; and 5) cingulate gyrus and parahippocampal gyrus.

\section{Discussion}

Given their phylogenetic and embryological development, especially the process of invagination of the surface of the brain, which effectively increases the cortical area without proportionally increasing the volume of the brain, ${ }^{64,79}$ the cerebral sulci, which delineate the respective gyri, can be considered natural extensions of the subarachnoid space. When they are deep and anatomically constant, they are referred to as fissures..$^{6,7}$ The principal sulci have approximate depths ranging from 1 to $3 \mathrm{~cm}$, and their walls harbor small gyri that face, adapt to, and connect with each other. Those gyri are generically designated the transverse gyri. The sulci that separate the transverse intrasulcal gyri vary in length and depth. At the surface of the brain, they become visible as incisures. The indentations caused by cortical arteries can have an appearance similar to that of the incisures.

To understand and correctly identify the sulci, and consequently the cerebral gyri, it is fundamental to consider the notion that the characterization of a given sulcus does not necessarily imply that it is composed of a single continuous space. A sulcus can consist of one or more parts, which in some cases can be oriented in different directions. Those parts can be long or short and can be isolated or connected to other sulci..$^{50}$

It is noteworthy that the timing of their embryological development and their degree of variability define ${ }^{11,46}$ a true morphological hierarchy, at the top of which are the fissures and principal sulci (Table 2). It is equally notable that this structural hierarchy is directly correlated with the functional importance of the areas to which the sulci are related, the more anatomically constant sulci being those that are topographically related to areas that are more specialized.

Typically, the sulci are divided into 4 types: limiting, axial, opercular, and complete..$^{79}$ Albeit complex, this system of classification is useful for demonstrating the heterogeneity of the cerebral sulci. The axial sulci develop along the axis of a functionally homogeneous area, as in the case of the posterior portion of the calcarine fissure, which is actually a fold situated in the center of the striate visual cortex. In any given gyrus, the invaginations made by the axial sulci result in the formation of subgyri, whose white matter can be designated subgyral sectors of the principal gyrus. The limiting sulci are situated between functionally different cortical areas. One example is the central sulcus, which separates the motor area from the sensory area. The opercular sulci are also situated between cortical areas that are structurally and functionally different. However, unlike the separations defined by the limiting sulci, those created by the opercular sulci exist only along their edges and not in their fundi. One example of an opercular sulcus is the lunate sulcus, which, when present, is oriented vertically, separating the striate and peristriate areas of the cortical surface and including the submerged parastriate area within its walls.

The sulci referred to as complete sulci are those whose fundi produce rises in the walls of the lateral ventricles. For example, the collateral sulcus creates the collateral eminence on the floor of the inferior horn, and the calcarine fissure causes the calcar avis in the medial wall of the posterior horn. ${ }^{79}$ This phenomenon has no functional significance and is only of morphological importance.

As some other authors have observed, the sylvian fissure and parietooccipital sulcus are the only sulci that do not fall into 1 of the 4 categories and therefore must be understood on the basis of their own development. ${ }^{50,79}$ The slower expansion of the insular cortex leads to its consequent submersion by the adjacent areas, and the convergence of those areas delineates the sylvian fissure, or lateral cerebral sulcus. The sylvian fissure is composed of a deep anterior and a posterior branch that harbor the sylvian cistern. The frontoparietal and temporal opercular surfaces constitute the walls of the sylvian fissure, and the insular surface is its floor.

The parietooccipital sulcus as well as the external perpendicular fissure that constitutes its extension over the superolateral surface is formed as a consequence of the development of the corpus callosum. Interhemispheric fibers originating in the occipital and temporal lobes are then carried by the posteriormost portion of the corpus callosum, which results in the invagination of the medial surface and the consequent creation of the parietooccipital sulcus. This process also leads to the development and grouping of smaller axial and limiting sulci that are located together in the intrasulcal walls of the parietooccipital sulcus.

From a more objective and practical standpoint and as proposed by Ono et al., ${ }^{50}$ the cerebral sulci can be characterized as follows: interrupted or continuous (sylvian fissure, calcarine fissure, callosal sulcus, parietooccipital sulcus, collateral sulcus, and central sulcus); and long or short. These authors divided the sulci into the following categories: long principal sulci (central, precentral, and postcentral sulci together with those listed above as continuous); short principal sulci (rhinal, olfactory, intraparietal, and intraoccipital, or occipital superior, sulci); short sulci composed of multiple branches (orbital and subparietal sulci); and free supplementary sulci (frontal medial and lunate sulci). ${ }^{50}$ Often the sulci are also composed of branches to which they are not connected or to which they are connected in an end-to-end, side-to-side, or endto-side manner, and such connections can even join 2 parallel sulci. Because of the frequency of such connections, there are discrepancies in the literature regarding the nomenclature and definitions of the sulci. ${ }^{14,50,72}$ To fully understand the cerebral sulci and gyri, one must realize that even the principal sulci can vary in form and size from 
person to person and that the surface of the brain constitutes a true continuum, presenting a serpentine configuration given its various forms of connections surrounding the sulcal extremities and running under the fundus of the sulci. ${ }^{82}$ The separation between neighboring and adjacent gyri is therefore only superficial and is structurally defined by the continuity and the fundi of the sulci that surround them. The interruption of a sulcus or the presence of a free sulcal extremity necessarily indicates the presence of a fold that connects different gyri or different sectors of the same gyrus. Each cerebral gyrus should therefore be understood as a region of the brain surface and not as an individual, anatomically well-defined neural structure. In terms of nomenclature, the gyri that are more rounded or quadrangular are usually referred to as lobules.

For microneurosurgical applications, is also notable that given the mechanism of invagination of the surface of the brain throughout its evolution and embryological development ${ }^{64}$ the sulci of the superolateral and inferior surfaces of the brain are consistently oriented toward the nearest ventricular cavity, which is especially evident in coronal slices of MR imaging studies. This disposition of the sulci is not seen on the medial surface of the hemispheres because the development of the sulci on this surface is directly related to that of the corpus callosum, and these sulci therefore tend to be arranged in parallel with this commissure. ${ }^{50}$

Considering that the cerebral gyri constitute a continuum via their multiple and, in part, variable superficial and deep connections, it is important to understand that the gyri are actually composed of arbitrarily circumscribed regions on the surface of the brain and that they are delineated by the cerebral sulci, which correspond to extensions of the subarachnoid space and are equally arbitrary in terms of their extensions and interruptions throughout the cerebral surface.

\section{Conclusions}

The brain fissures and sulci constitute the fundamental anatomical delimiting landmarks of the cerebral gyri and the main surgical corridors of modern microneurosurgery. Despite their variability, both cerebral sulci and gyri are organized according to a basic configuration that permits their identification.

\section{Disclosure}

The author reports no conflict of interest concerning the materials or methods used in this study or the findings specified in this paper.

\section{References}

1. Berger MS, Cohen WA, Ojemann GA: Correlation of motor cortex brain mapping data with magnetic resonance imaging. J Neurosurg 72:383-387, 1990

2. Boling W, Olivier A, Bittar RG, Reutens D: Localization of hand motor activation in Broca's pli de passage moyen. J Neurosurg 91:903-910, 1999

3. Broca P: Diagnostic d'un abcés situé au niveau de la région du langage, trépanation de cet abcès. Rev d'Anthrop 5:244-248, 1876, cited in Gusmão S, Silveira RL, Cabral G: [Broca and the birth of modern surgery.] Arq Neuropsiquiatr 58:11491152, 2000 (Portuguese)

4. Broca P: Remarques sur le siège de la facultè du langage articulé: suiries d'une observation d'aphémie (perte de la perole). Bull Soc d'Anth (Paris) 6:330-357, 397-407, 1861, cited in Finger S: Origins of Neuroscience. New York: Oxford University Press, 1994

5. Broca P: Sur la cinconvolution limbique et al scissure limbique. Bull Soc d'Anth 12:646-657, 1877, cited in Finger S: Origins of Neuroscience. New York: Oxford University Press, 1994

6. Broca P: Sur la topographie crânie-cerébrale ou sur les rapports anatomiques du crâne et du cerveau. Rev d'Anthrop 5:193-248, 1876

7. Broca P: Sur les rapports anatomiques des divers points de la surface du crâne et des diverses parties des hémisfères cérébraux. Bull Soc d'Anth 2:340, 1861, cited in Gusmão S, Silveira RL, Cabral G: [Broca and the birth of modern surgery.] Arq Neuropsiquiatr 58:1149-1152, 2000 (Portuguese)

8. Brodal A: Neurological Anatomy in Relation to Clinical Medicine, ed 3. New York: Oxford University Press; 1981

9. Brodmann K: Verleichende Lokalisations Lehre der Grosshirnrinde in Ihren Prinzipien dar Gestellt auf Grund des Zellenbanes. Leipzig: J. A. Barth, 1909 (reprinted 1925), Vol 12, cited in Penfield W: Cerebral Cortex of Man. New York: Macmillan, 1952

10. Butler AB, Hodos W: Comparative Vertebrate Neuroanatomy: Evolution and Adaptation, ed 2. Hoboken: John Wiley \& Sons, 2005

11. Chi JG, Dooling EC, Gilles FH: Gyral development of the human brain. Ann Neurol 1:86-93, 1977

12. Cianchi M, Breschi G: Leonardo Anatomia. Firenze: Giuti Gruppo Editoriale, 1997

13. Clarke E, Dewhurst K: Histoire Illustrée de la Fonction Cérébrale. Paris: Les Éditions Roger Dacosta, 1975

14. Duvernoy HM: The Human Brain. Wien: Springer, 1991

15. Duvernoy MH: The Human Hippocampus, ed 2. Berlin: Springer, 1998

16. Ebeling U, von Cramon D: Topography of the uncinate fascicle and adjacent temporal fiber tracts. Acta Neurochir (Wien) 115:143-148, 1992

17. Ebeling U, Eisner W, Gutbrod K, Ilmberger I, Schmid UD, Reulen HJ: Intraoperative speech mapping during resection of tumors in the posterior dominant temporal lobe. J Neurol 369:104, 1992

18. Excerpta Medica Foundation: Nomina Anatomica, ed 4. Amsterdam: Excerpta Medica, 1975

19. Excerpta Medica Foundation: Nomina Anatomica, ed 6. Amsterdam: Excerpta Medica, 1980

20. Excerpta Medica Foundation: Nomina Anatomica (PNA). Amsterdam: Excerpta Medica, 1955

21. Federative Committee on Anatomical Terminology: International Anatomical Terminology. Stuttgart: Thieme, 1998

22. Ferreira AG: [Latin-Portuguese Dictionary.] Porto: Porto Editora, 1966 (Portuguese)

23. Finger S: Origins of Neuroscience. New York: Oxford University Press, 1994

24. Gould SJ: The Book of Life. New York: WW Norton, 2001

25. Gould SJ: The Structure of the Evolutionary Theory. Cambridge: The Belknap Press of the Harvard University Press, 2002

26. Graña F, Rocca ED, Graña LR: Las Trepanaciones Craneanas en el Peru en la Época Pre-hispanica. Lima: Imprensa Santa Maria, 1954

27. Gratiolet LP: Memoire Sur Les Plis Cerébraux de L'homme et des Primates. Paris: Bertrand, 1854, cited in Tamraz JC, Comair YG: Atlas of Regional Anatomy of the Brain Using MRI. Berlin: Springer, 2000

28. Harkey HL, al-Mefty O, Haines DE, Smith RR: The surgi- 
cal anatomy of the cerebral sulci. Neurosurgery 24:651-654, 1989

29. Heimer L: The Human Brain and Spinal Cord: Functional Neuroanatomy and Dissection Guide, ed 2. New York: Springer Verlag, 1995

30. Heimer L: A new anatomical framework for neuropsychiatric disorders and drug abuse. Am J Psychiatry 160:1726-1739, 2003

31. Heimer L, Van Hoesen GW: The limbic lobe and its output channels: implications for emotional functions and adaptive behavior. Neurosci Biobehav Rev 30:126-147, 2006

32. Heimer L, Switzer RC, Van Hoesen GV: Ventral striatum and ventral pallidum. Trends Neurosci 5:83-87, 1982

33. Heimer L, Van Hoesen GW, Trimble M, Zahm DS: Anatomy of Neuropsychiatry: The New Anatomy of the Basal Forebrain and Its Implications for Neuropsychiatric Illness. Amsterdam: Elsevier, 2008

34. His W: Anatomishe Nomenklatur (Nomina Anatomica): Leipeiz: Verlag von Veit, 1895

35. Kluger $\mathbf{J}$ (ed): The landscape of the mind, in Time: Your Brain, A User's Guide. New York: Time Magazine, 2009

36. Lockard I: Desk Reference for Neuroanatomy: A Guide to Essential Terms. New York: Springer-Verlag, 1977

37. Ludwig E, Klinger J: Atlas Cerebri Humani. Basel: S. Karger, 1956

38. Lyons AS, Petrucelli RJ: Medicine, an Illustrated History. New York: Abradale Press, 1978

39. Martin JH: Neuroanatomy, Text and Atlas, ed 2. Stanford: Appleton and Lange, 1996

40. McGinty JF (ed): Advancing From the Ventral Striatum to the Extended Amygdala: Implications for Neuropsychiatry and Drug Abuse. Annals of the New York Academy of Sciences, Vol 877. New York: The New York Academy of Sciences, 1999

41. Mclean PD: A Triune Concept of the Brain and Behavior. Toronto: University of Toronto Press, 1973

42. Mello LE, Villares J, Leckman JF: Neuroanatomy of the basal ganglia. Psychiatr Clin North Am 20:691-704, 1997

43. Mesulam MM: Patterns in behavioral neuroanatomy: association areas, the limbic system, and hemispheric specialization, in Principles of Behavioral Neurosurgery. Philadelphia: F. A. Davis, 1987, pp 1-70

44. Naidich TP, Valavanis AG, Kubik S: Anatomic relationships along the low-middle convexity: Part I-Normal specimens and magnetic resonance imaging. Neurosurgery 36:517-532, 1995

45. Nieuwenhuys R, Voogt J, Van Huijzen C: The Human Central Nervous System, ed 3. Berlin: Springer-Verlag, 1988

46. Nishikuni K: [Study of the fetal and post-natal morphological development of the sulci of the brain] (thesis). São Paulo: Faculdade de Medicina, Universidade de São Paulo, 2006 (Portuguese)

47. Ojemann G, Ojemann J, Lettich E, Berger M: Cortical language localization in left, dominant hemisphere. An electrical stimulation mapping investigation in 117 patients. J Neurosurg 71:316-326, 1989

48. Olmos JS: The amygdaloid projection field in the rat brain as studied with the cupric-silver method, in Eleftheriou BE (ed): The Neurobiology of the Amygdala. New York: Plenum Press, 1972, pp 145-204

49. Olmos JS, Heimer L: The concept of ventral striatopallidal system and extended amygdala. Ann N Y Acad Sci 877:1-32, 1999

50. Ono M, Kubik S, Abernathey CD: Atlas of Cerebral Sulci. Stuttgart: Thieme, 1990

51. Penfield W, Rasmussen T: The Cerebral Cortex of Man. New York: Macmillan, 1952

52. Pia HW: Microsurgery of gliomas. Acta Neurochir (Wien) 80:1-11, 1986
53. Quiñones-Hinojosa A, Ojemann SG, Sanai N, Dillon WP, Berger MS: Preoperative correlation of intraoperative cortical mapping with magnetic resonance imaging landmarks to predict localization of the Broca area. J Neurosurg 99:311-318, 2003

54. Rasmussen T: Cortical resection for medically refractory focal epilepsy: results, lessons and questions, in Rasmussen $\mathrm{T}$, Marino R Jr (ed): Functional Neurosurgery. New York: Raven Press, 1979, pp 253-269

55. Rasmussen T: Surgery for central, parietal and occipital epilepsy. Can J Neurol Sci 18 (4 Suppl):611-616, 1991

56. Rasmussen T: Tailoring of cortical excisions for frontal lobe epilepsy. Can J Neurol Sci 18 (4 Suppl):606-610, 1991

57. Rhoton AL Jr: Cranial anatomy and surgical approaches. Neurosurgery 53:1-746, 2003

58. Ribas GC: [Considerations about the nervous system phylogenetic evolution, behavior, and the emergence of consciousness.] Rev Bras Psiquiatr 28:326-338, 2006 (Portuguese)

59. Ribas GC: [Neuroanatomical basis of behavior: history and recent contributions.] Rev Bras Psiquiatr 29:63-71, 2007 (Portuguese)

60. Ribas GC: [Surgical anatomy of microneurosurgical sulcal key-points] (thesis). São Paulo: Faculdade de Medicina, Universidade de São Paulo, 2005 (Portuguese)

61. Ribas GC, Ribas EC, Rodrigues CJ: The anterior sylvian point and the suprasylvian operculum. Neurosurg Focus 18(6):E2, 2005

62. Ribas GC, Yasuda A, Ribas EC, Nishikuni K, Rodrigues Junior AJ: Surgical anatomy of microneurosurgical sulcal key-points. Neurosurgery 59(ONS Suppl 4):ONS177-ONS209, 2006

63. Rolando L: Della Strutura degli Emisferi Cerebrali. Turin: Memorie de Regia Accademia delle Scienze di Torino, 1829, cited in Türe U, Yaşargil MG, Friedman AH, et al: Fiber dissection technique: lateral aspect of the brain. Neurosurgery 47:417-427, 2000

64. Sarnat HB, Netsky MG: Evolution of the Nervous System, ed 2. New York: Oxford University Press, 1981

65. Saunders JBCM, O'Malley CD: The Illustrations From the Works of Andreas Vesalius of Brussels. Cleveland: World, 1950

66. Sociedade Brasileira de Anatomia: [Anatomical Terminology.] São Paulo: Manole, 2001 (Portuguese)

67. Squire LR, Bloom FE, McConnell SK, Roberts JL, Spitzer NC, Zigmond MJ: Fundamental Neuroscience, ed 2. Amsterdam: Academic Press, 2003

68. Stieve H: Nomina Anatomica, ed 4. Jena: Verlag von Gustav Fisser, 1949

69. Tamraz JC, Comair YG: Atlas of Regional Anatomy of the Brain Using MRI. Berlin: Springer, 2000

70. Taveras JM, Wood EH: Diagnostic Neuroradiology, ed 2. Baltimore: Williams and Wilkins, 1976, Vol 1

71. Testut L, Jacob O: [Topographic Anatomy Textbook, ed 5.] Barcelona: Salvat, 1932 (Spn)

72. Testut L, Latarjet A: [Human Anatomy Textbook, ed 8.] Barcelona: Salvat, 1932 (Spn)

73. Türe U, Yaşargil DCH, Al-Mefty O, Yaşargil MG: Topographic anatomy of the insular region. J Neurosurg 90:730-733, 1999

74. Türe U, Yaşargil MG, Friedman AH, Al-Mefty O: Fiber dissection technique: lateral aspect of the brain. Neurosurgery 47:417-427, 2000

75. Uematsu S, Lesser R, Fisher RS, Gordon B, Hara K, Krauss GL, et al: Motor and sensory cortex in humans: topography studied with chronic subdural stimulation. Neurosurgery 31:59-72, 1992

76. Vieussens R: Nevrographia Universalis. Frankfurt: Georgium Wilmelmum Künnium, 1690

77. Von Economo C: Cellular Structure of the Human Cerebral Cortex. Triarhou LC, trans. Basel: Karger, 2009 
78. Wen HT, Rhoton AL Jr, de Oliveira E, Cardoso ACC, Tedeschi $\mathrm{H}$, Baccanelli M, et al: Microsurgical anatomy of the temporal lobe: part 1: mesial temporal lobe anatomy and its vascular relationships as applied to amygdalohippocampectomy. Neurosurgery 45:549-592, 1999

79. Williams PL, Warwick R (ed): Gray's Anatomy, ed 36. Philadelphia: Saunders, 1980

80. Yaşargil MG: A legacy of microneurosurgery: memoirs, lessons, and axioms. Neurosurgery 45:1025-1092, 1999

81. Yaşargil MG: Microneurosurgery. Stuttgart: Georg Thieme, 1984, Vol 1

82. Yaşargil MG: Microneurosurgery. Stuttgart: Georg Thieme, 1994, Vol 4a

83. Yaşargil MG: Microneurosurgery. Stuttgart: Georg Thieme, 1996, Vol 4b

84. Yaşargil MG, Cravens GF, Roth P: Surgical approaches to "inaccessible" brain tumors. Clin Neurosurg 34:42-110, 1988
85. Yaşargil MG, Kasdaglis K, Jain KK, Weber HP: Anatomical observations of the subarachnoid cisterns of the brain during surgery. J Neurosurg 44:298-302, 1976

86. Yaşargil MG, Krisht AF, Türe U, Al-Mefty O, Yaşargil DCH: Microsurgery of insular gliomas: Part I: surgical anatomy of the Sylvian cistern. Contemp Neurosurg 24:1-8, 2002

87. Yaşargil MG, Teddy PJ, Roth P: Selective amygdalo-hippocampectomy. Operative anatomy and surgical technique. Adv Tech Stand Neurosurg 12:93-123, 1985

Manuscript submitted October 19, 2009.

Accepted November 10, 2009.

Address correspondence to: Guilherme Carvalhal Ribas, M.D., Rua Eduardo Monteiro, 567, São Paulo, Brazil, 05614-120. email: guilherme@ribas.med.br. 